\title{
AKT isoforms modulate Th1-like Treg generation and function in human autoimmune disease
}

\author{
Alexandra Kitz ${ }^{\dagger}$, Marine de Marcken ${ }^{\dagger}$, Anne-Sophie Gautron, Mitja Mitrovic, David A Hafler ${ }^{\ddagger}$ \& \\ Margarita Dominguez-Villar ${ }^{*}$ *
}

\begin{abstract}
Foxp $3^{+}$regulatory $\mathrm{T}$ cells (Tregs) exhibit plasticity, which dictates their function. Secretion of the inflammatory cytokine IFN $\gamma$, together with the acquisition of a T helper 1 (Th1)-like effector phenotype as observed in cancer, infection, and autoimmune diseases, is associated with loss of Treg suppressor function through an unknown mechanism. Here, we describe the signaling events driving the generation of human Th1-Tregs. Using a genome-wide gene expression approach and pathway analysis, we identify the PI3K/AKT/Foxo1/3 signaling cascade as the major pathway involved in IFN $\gamma$ secretion by human Tregs. Furthermore, we describe the opposing roles of AKT isoforms in Th1-Treg generation ex vivo. Finally, we employ multiple sclerosis as an in vivo model with increased but functionally defective Th1-Tregs. We show that the $\mathrm{PI}$ IK/AKT/Foxo1/3 pathway is activated in ex vivo-isolated Tregs from untreated relapsing-remitting MS patients and that blockade of the pathway inhibits IFN $\gamma$ secretion and restores the immune suppressive function of Tregs. These data define a fundamental pathway regulating the function of human Tregs and suggest a novel treatment paradigm for autoimmune diseases.
\end{abstract}

Keywords autoimmunity; Foxp3; IFN $\gamma$; plasticity; Tregs

Subject Categories Immunology; Molecular Biology of Disease; Signal Transduction

DOI 10.15252/embr.201541905 | Received 10 December 2015 | Revised 4 May

2016 | Accepted 13 May 2016 | Published online 16 June 2016

EMBO Reports (2016) 17: 1169-1183

\section{Introduction}

$\mathrm{CD} 4{ }^{+} \mathrm{CD} 25^{\text {high }} \mathrm{CD} 127^{\text {low }}$ regulatory $\mathrm{T}$ cells (Tregs) are essential in controlling self-reactivity and immune homeostasis [1,2]. Foxp3 is the master regulator transcription factor of this lineage, and its expression is important for Treg development, maintenance, and function $[1,3]$. It has been shown that Tregs can produce IL-17 [4,5] and IFN $\gamma$ in murine and human systems [6-10] and IFN $\gamma$-secreting $\mathrm{CD} 4{ }^{+} \mathrm{CD} 25^{\text {hi }} \mathrm{CD} 127^{\text {low/neg }}$ Foxp $3^{+}$regulatory T cells (Th1-Tregs) are increased in frequency in autoimmune diseases [6,9] and infection [10]. We previously demonstrated that Tregs isolated ex vivo from patients with relapsing-remitting multiple sclerosis (RRMS) are defective in function and contain an increased frequency of Th1Tregs. IFN $\gamma$ blockade in ex vivo co-culture assays significantly improved their suppressive capacity, suggesting that IFN $\gamma$ secretion by Tregs is part of the defect in immune regulation observed in patients with MS [11-13]. Similar results have been observed in patients with type 1 diabetes [9].

In vitro, human Tregs can also acquire a Th1 phenotype when stimulated in the presence of IL-12. This phenotype is characterized by the upregulation of the master regulator of T helper 1 (Th1) cells, T-bet, and some other Th1 markers such as CXCR3 and CCR5, while maintaining Foxp3 expression [6,9]. IL-12-induced human Th1Tregs display a defect in suppression as compared to IFN $\gamma^{-}$Tregs, although they still retain a degree of suppressive capacity ex vivo $[6,9]$. Although Th1-Tregs have been observed in different disease settings, the molecular mechanisms and signaling pathways that are involved in their generation and function have not been elucidated, which is a critical step for devising specific therapies to correct losses of immune tolerance observed in autoimmune diseases.

Here, we describe the signaling pathway components that are responsible for Th1-Treg generation and function in humans. By using a genome-wide gene expression approach and pathway analysis, we defined the PI3K/AKT/Foxol/3 as the major pathway involved in IFN $\gamma$ secretion by Tregs. Using an in vitro model of Th1Treg generation, we confirmed that this pathway is activated during Th1-Treg reprogramming and its modulation by either activation of PI3K or AKT1 or inhibition of PTEN or Foxo1/3 is sufficient for human Tregs to acquire a Th1 phenotype. We further demonstrate the specific and non-overlapping roles of the three AKT isoforms in Th1-Treg generation, demonstrating that while AKT1 inhibition diminishes IFN $\gamma$ secretion, AKT3 silencing is sufficient to induce the Th1 phenotype. The physiological relevance of our observation is confirmed by data demonstrating that the PI3K/AKT/Foxol/3 pathway is activated in Tregs from untreated RRMS patients as compared to healthy donors in vivo, and blockade of this pathway inhibits IFN $\gamma$ secretion by MS Tregs and restores the defective suppressive function. These data define a fundamental pathway

\footnotetext{
Departments of Neurology and Immunobiology, Yale School of Medicine, New Haven, CT, USA

*Corresponding author. Tel: +1 203737 3399; E-mail: margarita.dominguez-villar@yale.edu

These authors contributed equally to this work as first authors

*These authors contributed equally to this work as last authors
} 
altered in the function of Tregs underlying autoimmune disease potentially amenable to immune targeting.

\section{Results}

\section{Ex vivo-isolated IFN $\gamma^{+}$Tregs possess a specific gene expression signature}

In order to examine signaling pathways differentially expressed in Th1-Tregs, $\mathrm{CD} 4^{+} \mathrm{CD} 25^{\text {hi }} \mathrm{CD} 127^{\text {low/neg }}$ Tregs from eight healthy individuals were sorted ex vivo and stimulated for $4 \mathrm{~h}$ with PMA and ionomycin to induce IFN $\gamma$ secretion. Tregs were sorted based on the expression of IFN $\gamma$ using an IFN $\gamma$ capture kit, and purity was confirmed by Foxp3 staining as well as IFNG and TBX21 gene expression (Fig EV1). A genome-wide microarray analysis was performed on IFN $\gamma^{+}$and IFN $\gamma^{-}$Treg populations; statistical analysis (see Materials and Methods, GEO accession number GSE71274) identified 4393 genes that were differentially expressed (DEG, $P$-value cutoff 0.05 and fold change cutoff 1.5). Ingenuity Pathway Analysis elucidated specific signaling pathways enriched in DEGs, listed by order of significance (Fisher's exact test, Appendix Table S1). The PI3K/AKT/Foxo1/3 pathway was the most significant, and Fig 1A shows the expression values of selected molecules from this pathway as fold increase/decrease in IFN $\gamma^{+}$Tregs as compared to IFN $\gamma^{-}$ Tregs. AKT1 and PHLPP2 were significantly upregulated, while AKT2, AKT3, PIK3CA, and PTEN were downregulated. The differential expression of these targets was confirmed by real-time PCR in a new set of IFN $\gamma^{+}$and IFN $\gamma^{-}$Tregs isolated from nine healthy individuals (Fig 1B).

To examine whether the PI3K/AKT/Foxo1/3 pathway was differentially expressed during Th1-Treg reprogramming, we stimulated Tregs ex vivo with anti-CD3 and anti-CD28 in the presence or absence of IL-12 and gene expression was examined at 24-h intervals for a total of 4 days [6] (Fig 1C). We observed a significant upregulation of AKT1 and PHLPP2 and a downregulation of AKT2, AKT3, FOXO1, FOXO3, and PTEN gene expression. PIK3CA showed a trend toward downregulation, but it did not reach statistical significance. Gene expression data was confirmed at protein level on IL-12-driven Th1-Tregs (Fig 1D and E). Interestingly, we observed differential protein expression of the three AKT isoforms, with a significant increase in AKT1 and decreased expression of AKT3 on Th1-Tregs as compared to Tregs (Fig 1E). Foxo3 and PTEN were both downregulated, while Foxol did not show a statistically significant difference in expression in Th1-Tregs as compared to Tregs (data not shown). These data demonstrate that the PI3K/AKT/ Foxo1/3 signaling pathway is differentially expressed during Th1Treg reprogramming.

\section{$\mathrm{PI3K/AKT/Foxo} \mathrm{pathway} \mathrm{is} \mathrm{activated} \mathrm{during} \mathrm{Th1-Treg} \mathrm{generation}$}

We next sought to examine whether the PI3K/AKT/Foxol/3 pathway was activated during generation of Th1-Tregs. Activation of this pathway starts by different stimuli that trigger a variety of receptors such as T-cell receptor (TCR) [14], co-stimulatory molecules such as CD28 [15], cytokine receptors [16], G protein-coupled receptors (GPCR) [17], and insulin [18] among others. This initial stimulation causes the phosphorylation and activation of PI3K, which then catalyzes the formation of the second messenger phosphatidylinositol-3,4,5-triphosphate (PIP3) [19]. PIP3 can then bind PDK1 and AKT, and AKT is activated by PDK1 by phosphorylation on residue Thr 308 [20], being able to phosphorylate different downstream targets. We hypothesized that during Th1-Treg reprogramming, IL-12 stimulation would activate PDK1, which in turn would phosphorylate AKT. In order to examine this hypothesis, we measured phosphorylation of AKT at Thr 308 after the addition of IL-12 for 50 min by FACS. IL-12 induced AKT phosphorylation as early as 20 min after stimulation (Fig 2A), and this increase was maintained for 20 additional minutes (Appendix Fig S1).

Once activated, AKT phosphorylates many downstream targets, among them the transcription factors Foxo1 and Foxo3 [21]. We thus examined the phosphorylation status of Foxo1 and Foxo3 upon IL-12 stimulation and observed that IL-12 induced a significant increase in the phosphorylation of both transcription factors as compared to Tregs treated with vehicle (Fig 2A). Interestingly, the increased phosphorylation of AKT, Foxo1, and Foxo3 was observed not only at short time points after IL-12 addition, but also $16 \mathrm{~h}$ after stimulation with anti-CD3 and anti-CD28 in the presence of IL-12 as compared to vehicle (Fig $2 \mathrm{~B}$ and $\mathrm{C}$ ), suggesting that the activation of the pathway is maintained during Th1-Treg reprogramming.

Foxo1 and Foxo3 reside in the nucleus of Tregs, where they help maintain Foxp3 expression and suppressive capacity [22]. When phosphorylated by AKT, they are excluded from the nucleus and thus not able to regulate their transcriptional targets [23]. We hypothesized that IL-12-driven phosphorylation of Foxo1 and Foxo3 would exclude them from the nucleus, thus preventing regulation of their transcriptional targets. Foxo1 positively regulates the expression of BCL2L11 [24], IL7R [25], and CTLA4 [22] and negatively regulates $P D X 1$ [26] expression while Foxo3 induces the expression of BNIP3 [27] and inhibits ID1 [28]. IL-12 stimulation induced the downregulation of BCL2L11, IL7R, and BNIP3, and the upregulation of $P D X 1$ and $I D 1$, suggesting that both transcription factors were not regulating their transcriptional targets (Fig 2D).

\section{Activation of the PI3K/AKT/Foxo pathway induces IFN $\gamma$ secretion and impairs the suppressive capacity of Tregs}

As the PI3K/AKT/Foxo1/3 pathway can be activated by engagement of different receptors, we hypothesized that direct activation of this pathway would be sufficient to induce the Th1-Treg phenotype independent of IL-12 or any other stimuli. To examine this hypothesis, we used a PTEN inhibitor (SF1670) [29], an AKT activator (SC-79) [30], a PI3K activator (740 Y-P) [31], and a Foxo1/3 inhibitor (AS1842856) [32]. Tregs were stimulated with anti-CD3, anti-CD28, and IL-2 in the presence of these PI3K/AKT/Foxo1/3 inhibitors/ activators, and we measured TBX21 and IFNG gene expression every $24 \mathrm{~h}$ for a total of 3 days (Fig 3A). The inhibitors/activators induced the predicted increase in IFNG and TBX21 gene expression based on our array data of Th1-Tregs. The increase in IFNG gene expression was confirmed at protein level by stimulating Tregs in the presence of the different activators for 3 days (Fig $3 \mathrm{~B}$ and $\mathrm{C}$ ). Interestingly, all small molecules reverted the anergic-like phenotype observed in Tregs, measured by proliferation and IL-2 secretion by Tregs after activation in the presence of the molecules (Appendix Fig S2). To confirm that activation of the PI3K/AKT/Foxo1/3 pathway induced the Th1-Treg phenotype, we pre-incubated sorted Tregs with either 

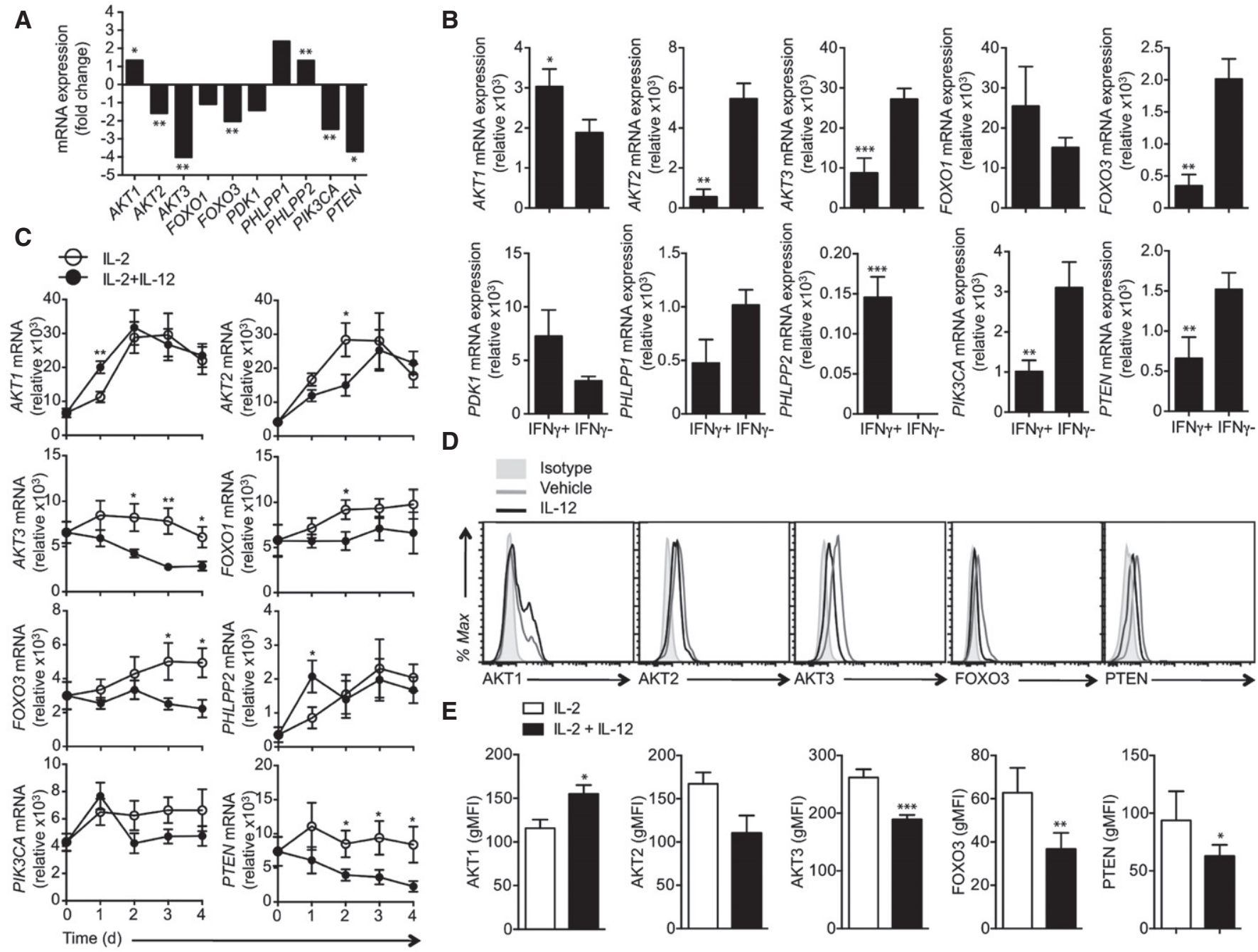

Figure 1. The PI3K/AKT/Foxo pathway is differentially expressed in Th1-Tregs.

A Fold change in mRNA expression of members of the PI3K/AKT/Foxo pathway in IFN $\gamma^{+}$as compared to IFN $\gamma^{-}$Tregs in the microarray analysis.

B mRNA expression of members of the PI3K/AKT/Foxo signaling pathway in sorted IFN $\gamma^{+}$and IFN $\gamma^{-}$Tregs.

C Sorted $\mathrm{CD} 4^{+} \mathrm{CD} 25^{\text {high }} \mathrm{CD} 127^{-}$Tregs were stimulated in the presence of IL-2 (white) or IL-2 + IL-12 (black dots), and RNA was isolated every $24 \mathrm{~h}$ for 4 days. mRNA expression of members of the PI3K/AKT/Foxo signaling pathway was analyzed.

D Representative examples of the protein expression of AKT1, AKT2, AKT3, PTEN, and FOXO3 on sorted CD4 ${ }^{+} \mathrm{CD} 25^{\text {high }} \mathrm{CD} 127^{-}$Tregs stimulated in the presence of IL-2 (gray line) or IL-2 + IL-12 (black line) and day 2 for AKT1, AKT2, AKT3, and PTEN and day 3 for FOXO3. Gray histogram represents the isotype.

E Statistical analysis of AKT1, AKT2, AKT3, FOXO3, and PTEN gMFI of $n=6$ experiments performed.

Data information: Data are presented as mean \pm s.e.m. ${ }^{*} P<0.05,{ }^{* *} P<0.005,{ }^{* \star *} P<0.0005$. $t$-test was performed for (B) and (E), and a two-way ANOVA corrected for multiple comparisons with a Sidak test for (C).

vehicle, SF1670, SC-79, or 740 Y-P for $1 \mathrm{~h}$, washed the cells to remove traces of the drugs, and co-cultured the Tregs with CFSElabeled responder $\mathrm{T}$ cells (Tresp) to examine their suppressive capacity (Fig 3D and E). While Tresp proliferation was significantly inhibited in the presence of Tregs pre-incubated with vehicle, Tregs that had been pre-incubated with either SF1670, SC-79, or 740 Y-P displayed a defect in their suppressive ability as compared to vehicle-treated Tregs (Fig 3D), which was observed at all Treg:Tresp ratios tested (Fig 3E).

The consequence of activation of the PI3K/AKT pathway by either activation of PI3K or AKT or inhibition of PTEN is the activation of Foxo1 and Foxo3, leading to their nuclear exclusion. Foxo1 and Foxo3 are thought to be important for Treg function and stability in mice [22], so we pre-incubated Tregs with different doses of the Foxo1/3 inhibitor AS184256 before co-culturing with CFSElabeled Tresp to measure their suppressive capacity (Fig 4A). Tregs pre-incubated with AS184256 displayed a decrease in suppressive function as compared to vehicle-treated Tregs in a dose-dependent manner, as the percentage of suppression was lower in those cells pre-treated with the highest concentration of AS184256 (Fig 4A and B). This defect was observed in all Treg:Tresp ratios examined, and it was not due to an effect on Treg viability, as there were no significant differences in the frequency of viable cells after treatment with AS184256 as compared to vehicle (data not shown). To examine 
A
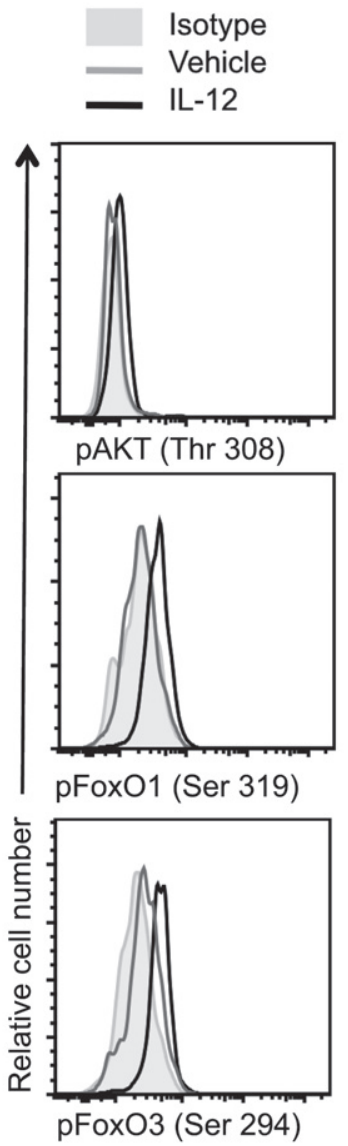

B
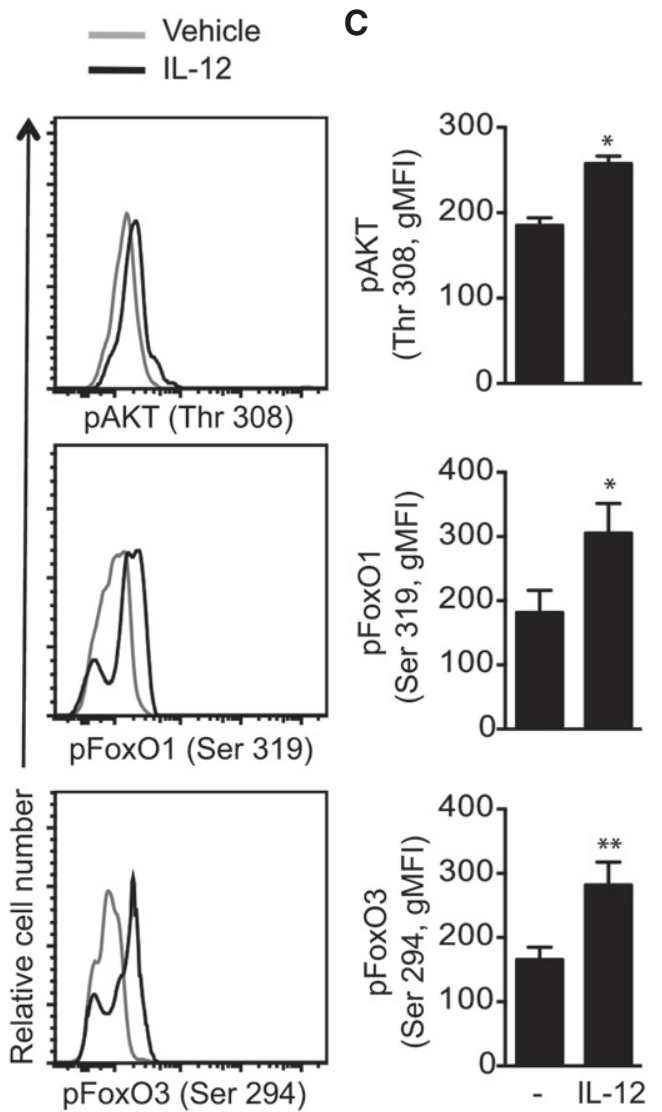

D
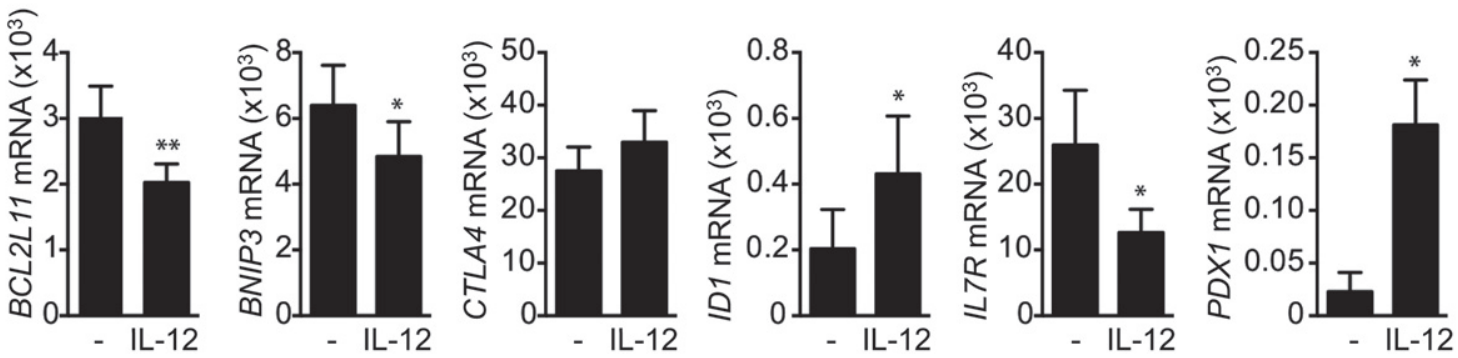

Figure 2. The PI3K/AKT/Foxo pathway is activated during Th1-Treg generation.

Sorted $\mathrm{CD} 4{ }^{+} \mathrm{CD} 25^{\text {hi }} \mathrm{CD} 127^{-}$Tregs were stimulated with anti-CD3, anti-CD28, and IL-2 in the presence or absence of IL- 12 .

A Tregs were stimulated for 50 min with IL-12 or vehicle. Histograms show a representative example of $n=8$ independent experiments performed of the expression of phosphorylated molecules at $30 \mathrm{~min}$ after stimulation for PAKT and pFox01, and at $40 \mathrm{~min}$ for pFox03 in vehicle-treated (gray open histogram) or IL-12-treated (dark open histogram) Tregs. Gray histograms represent the isotype. The time points selected were based on kinetic experiments performed for a total of 50 min.

B Tregs were stimulated in the presence (black) or absence (gray) of IL-12, and the expression of phosphorylated molecules was examined $16 \mathrm{~h}$ later.

C gMFI of pAKT, pFoxo1, and pFoxo3 of $n=6$ independent experiments performed.

D Foxo1 and Fox03 transcriptional targets expression on sorted CD4 ${ }^{+} C D 25^{\text {hi }} C D 127^{-}$Tregs stimulated in the presence of vehicle or IL-12 at day 2 after stimulation $(n=8)$

Data information: Data are presented as mean \pm s.e.m. ${ }^{*} P<0.05$, ${ }^{* *} P<0.005$. $t$-test was performed for (C) and (D).

whether the defect in suppression observed was due to the increase in IFN $\gamma$ secretion by Tregs, as was the case in IL-12-driven Th1-Tregs [6], we examined the frequency of IFN $\gamma$-secreting Tregs in the co-cultures with Tresp after being pre-incubated with AS1842856. For this purpose, after 4 days in co-culture, we added GolgiStop to the cultures for $6 \mathrm{~h}$ and we stained for IFN $\gamma$ and Foxp3 (Fig 4C). At the time point examined, there was a significant increase in the frequency of IFN $\gamma^{+} \mathrm{Foxp}^{+}$Tregs in co-cultures in which Tregs had been pre-incubated with AS1842856 as compared to vehicle-treated Tregs (Fig 4C and Appendix Fig S3). This increase was dose-dependent, as the frequency of IFN $\gamma^{+} \mathrm{Foxp}^{+}$Tregs was higher in co-cultures where Tregs had been pre-incubated with higher doses of AS1842856 (Fig 4C and D). Moreover, we found a statistically significant inverse correlation between the percentage of 

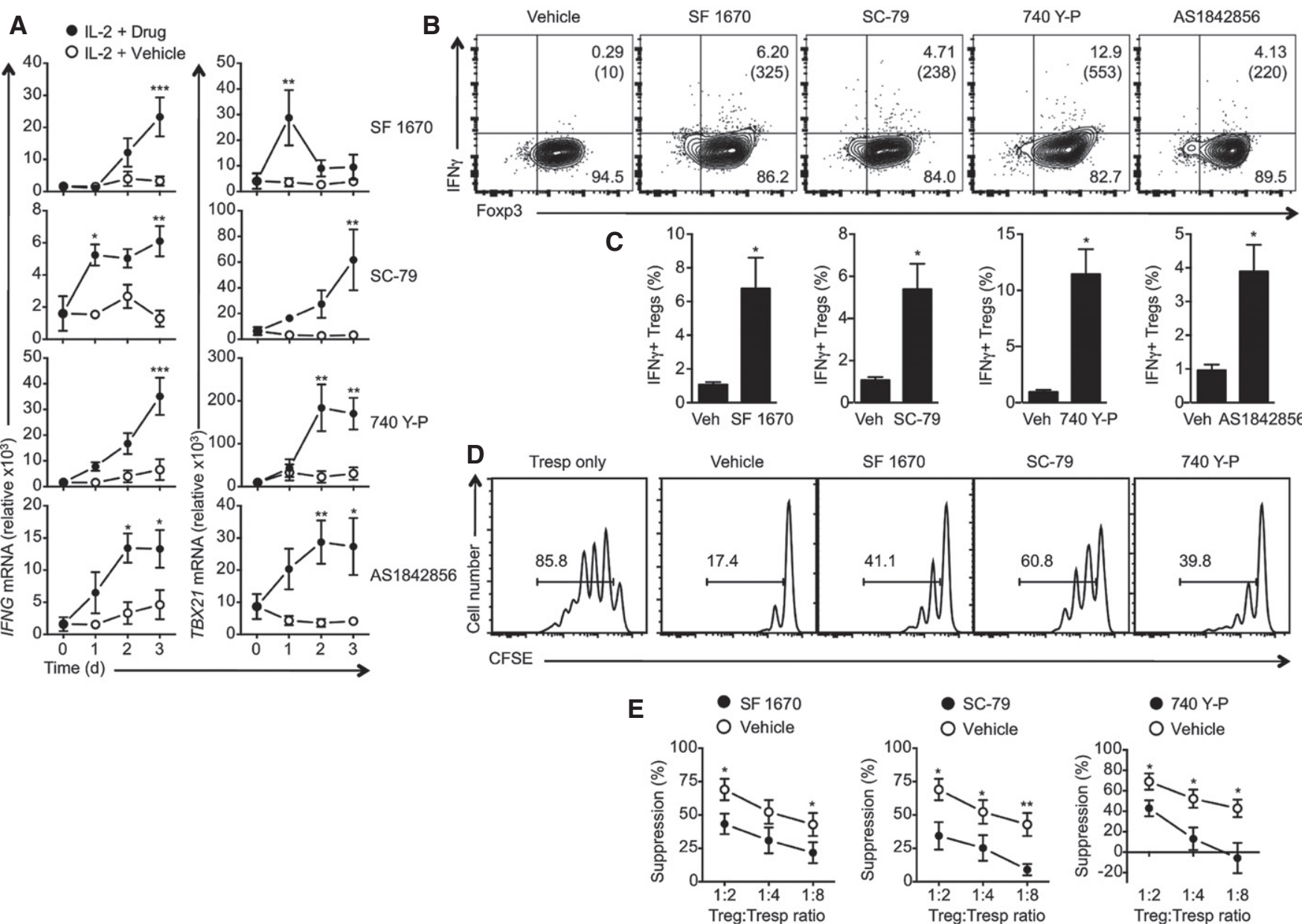

Figure 3. Activation of the PI3K/AKT/Foxo pathway is sufficient to induce the Th1 phenotype on Tregs.

A Sorted CD4 ${ }^{+} C D 25^{\text {hi }} C D 127^{-}$Tregs were stimulated in the presence of vehicle (white dots) or either SF1670 (first row), SC-79 (second row), 740 Y-P (third row), or AS1842856 (fourth row, black dots) for 3 days, and IFNG and TBX21 gene expression was examined every $24 \mathrm{~h}$. Statistical analysis of $n=4$ independent experiments performed.

B Representative example of the frequency of IFN $\gamma^{+} \mathrm{Foxp}^{+}$cells in Tregs stimulated in the presence of vehicle, SF1670, SC-79, 740 Y-P, or AS1842856 for 3 days after $4 \mathrm{~h}$ of stimulation with PMA and ionomycin. Numbers in parentheses represent total cell numbers.

C Statistical analysis of the frequency of IFN $\gamma^{+}$Foxp3 ${ }^{+}$cells in Tregs stimulated in the presence of vehicle, SF1670, SC-79, 740 Y-P, or AS1842856 of $n=5$ experiments performed.

D Proliferation of CFSE-labeled responder T cells alone (left histogram) or co-cultured with Tregs that have been pre-incubated with either vehicle, SF1670, SC-79, or 740 Y-P at day 4 after co-culture at a 1:2 Treg:Tresp ratio.

E Statistical analysis of the percentage of suppression at different Treg:Tresp ratios of $n=6$ experiments performed.

Data information: Data are presented as mean \pm s.e.m. ${ }^{*} P<0.05,{ }^{\star *} P<0.005,{ }^{* \star *} P<0.0005$. Two-way ANOVA corrected for multiple comparisons with a Sidak test was performed for (A) and (E), and a $t$-test for (C).

suppression and the frequency of IFN $\gamma^{+} \mathrm{Foxp}^{+}$in the co-cultures, suggesting that the higher the frequency of IFN $\gamma^{+} \mathrm{Foxp}^{+}$Tregs in co-culture, the higher the defect in suppressive capacity (Fig 4E).

To confirm these results, we used shRNAs specific for Foxo1 and Foxo3 to silence both proteins in Tregs (Fig EV2) and measured IFNG and TXB21 gene expression. Both Foxo1 silencing and Foxo3 silencing were sufficient to induce a significant increase in IFNG and TBX21 gene expression as compared to non-target-transduced Tregs (Fig 4F). Moreover, Foxo1- and Foxo3-silenced Tregs were defective in suppressing the proliferation of Tresp in in vitro suppression assays (Fig 4G). These data suggest that activation of the PI3K/ AKT/Foxo1/3 pathway is sufficient to induce the Th1-Treg phenotype on human Tregs and that both Foxo1 and Foxo3 play overlapping roles in human Th1-Treg generation.

\section{Differential role of AKT isoforms on Th1-Treg generation}

Ex vivo-isolated and in vitro-generated Th1-Tregs expressed increased levels of AKT1 and decreased levels of AKT2 and AKT3 (Fig 1), suggesting that the three isoforms may be performing non-overlapping roles in Th1-Treg generation. To examine this hypothesis, we silenced AKT1, AKT2, and AKT3 in Tregs with specific shRNA or non-target control (Fig EV3) and we examined IFNG, TBX21, FOXP3, and IL10 expression, as we 

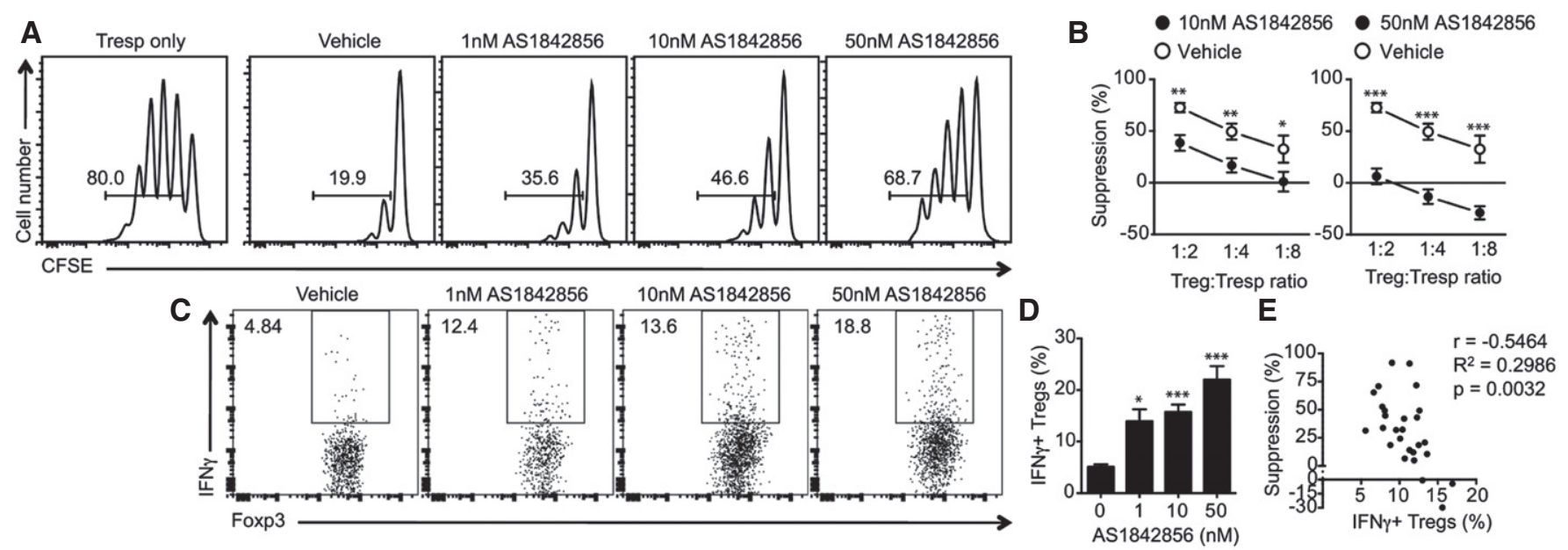

$\mathbf{F}$

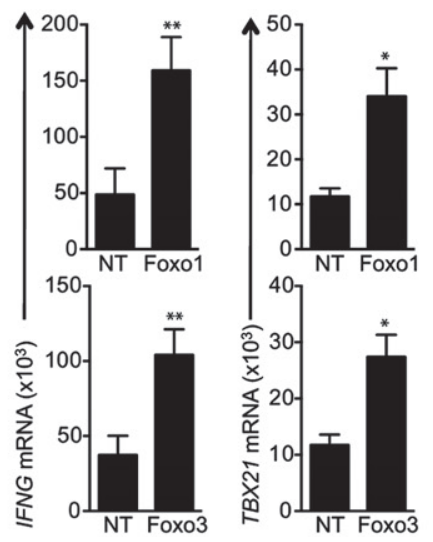

G
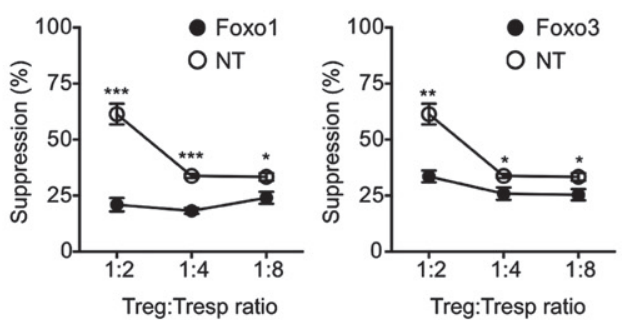

Figure 4. Foxo1/3 inhibition is sufficient to induce the Th1 phenotype on Tregs.

A Sorted $\mathrm{CD} 4{ }^{+} \mathrm{CD} 25^{\text {hi }} \mathrm{CD} 127^{-}$were pre-incubated with increasing concentrations of AS1842856, washed, and co-cultured with CFSE-labeled responder T cells. Representative histograms of proliferation of Tresp alone (left) or in co-culture with Tregs treated with either vehicle or different concentrations of AS1842856 measured at day 4 at a 1:2 Treg:Tresp ratio.

B Statistical analysis of the suppression of proliferation at different Treg:Tresp ratios $(n=6)$ on Tregs pretreated with vehicle (white dots) or $10 \mathrm{nM}$ (black dots, left diagram) or $50 \mathrm{nM}$ AS1842856 (black dots, right diagram).

C Representative dot plots of the frequency of IFN $\gamma^{+}$Foxp $^{+}$Tregs in the co-cultures in (A) at a 1:2 Treg:Tresp ratio after adding GolgiStop for the last 6 h.

D Statistical analysis of the frequency of IFN $\gamma^{+} \mathrm{Foxp}^{+}$Tregs in the co-cultures in (A) of $n=6$ experiments performed.

E Correlation between frequency of IFN $\gamma^{+}$Foxp $^{+}$Tregs in co-cultures and percentage of suppression in Tregs pre-incubated with different doses of AS1842856 at a 1:4 Treg:Tresp ratio.

F IFNG and TBX21 gene expression on Tregs transduced with a Foxo1 or Fox03 shRNA as compared to the NT-shRNA control, measured at day 6 after 4 h of stimulation with PMA and ionomycin.

G Percentage of suppression of CFSE-labeled Tresp proliferation co-cultured with Tregs transduced with a non-target (white dots), Foxol (left diagram, black dots), or Fox03 shRNA (right diagram, black dots) at different Treg:Tresp ratios $(n=4)$.

Data information: Data are presented as mean \pm s.e.m. ${ }^{*} P<0.05$, ${ }^{* \star} P<0.005,{ }^{* \star *} P<0.0005$. Two-way ANOVA corrected for multiple comparisons with a Sidak test was performed for (B) and (C), a one-way ANOVA with a Tukey's post-test for (D) and a $t$-test for (F). For correlation in (E), $r$ was calculated by Pearson correlation coefficient.

had previously shown that Th1-like Tregs expressed increased levels of IL-10 as compared to Tregs [6]. AKT1-silenced Tregs expressed decreased levels of IFNG and TBX21 as compared to the non-target shRNA, with no effect on IL10 expression. In contrast, AKT3 silencing significantly upregulated IFNG, IL10, and TBX21 expression. We did not observe any effect of AKT2 silencing on Th1-Treg-specific gene expression, and FOXP3 expression was not altered by AKT silencing (Fig 5A).

AKT-silenced Tregs were then stimulated in the presence of IL-12 or vehicle. Interestingly, AKT1-silenced Tregs showed decreased frequency while AKT3-silenced Tregs increased frequency of IFN $\gamma^{+} \mathrm{Foxp}^{+}$as compared to non-target-transduced Tregs. AKT2silenced cells did not show an alteration in the frequency of IFN $\gamma^{+}$Foxp $^{+}$Tregs. Similarly, when stimulated in the presence of IL-12, AKT1-silenced Tregs were defective in generating Th1-Tregs, in contrast to AKT3-silenced Tregs. AKT2-silenced Tregs also showed a defect in IL-12 response, displaying a lower frequency of IFN $\gamma^{+}$Foxp $^{+}$Tregs than non-target control Tregs (Fig 5B and C).

We next examined the suppressive capacity of AKTsilenced Tregs in co-cultures with Tresp (Fig 5D and E). While 

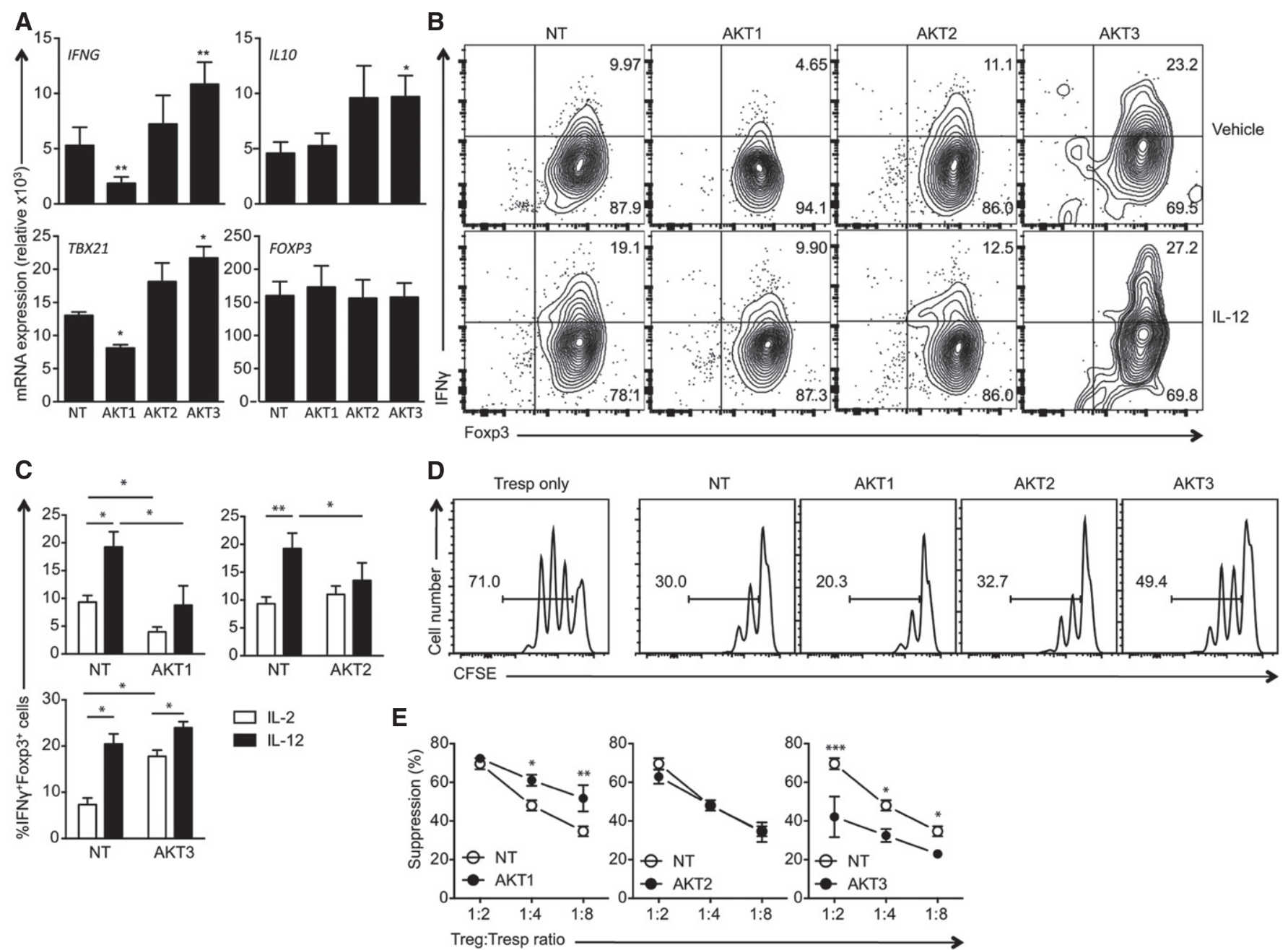

Figure 5. Differential roles of AKT isoforms in Th1-Treg generation.

Sorted Tregs were stimulated with anti-CD3, anti-CD28, IL-2, and shRNA specific for AKT1, AKT2, AKT3, or non-target control and resorted based on GFP expression at day 5 .

A IFNG, TBX21, IL10, FOXP3 gene expression after sort at day $5(n=5)$.

B Representative example of staining on resorted Tregs stimulated with PMA and ionomicin for 4 h at day $5(n=4)$.

C Statistical analysis of the frequency of IFN $\gamma^{+}$Foxp $3^{+}$Tregs after AKT silencing $(n=4)$.

D shRNA-transduced cells were co-cultured with CFSE-labeled Tresp, and Tresp proliferation was measured after 4 days.

E Statistical analysis of the percentage of suppression by AKT1-, AKT2-, and AKT3-silenced Tregs as compared to NT-transduced cells (white dots) of $n=6$ experiments performed.

Data information: Data are presented as mean \pm s.e.m. ${ }^{\star} P<0.05,{ }^{* \star} p<0.005,{ }^{* \star *} p<0.0005$. One-way ANOVA with a Tukey's post-test was performed for (A), and a two-way ANOVA with correction for multiple comparisons for (C) and (E).

non-target-transduced Tregs efficiently suppressed Tresp proliferation, AKT3-silenced Tregs were defective in Tresp inhibition. We did not observe any difference in suppression between control and AKT2-silenced Tregs, but AKT1-silenced Tregs showed an increase in their suppressive capacity as compared to the control. These data suggest that AKT isoforms play non-overlapping roles on Th1-Treg generation.

\section{Blockade of PI3K/AKT/Foxo1/3 pathway on Th1-Tregs inhibits IFN $\gamma$ secretion and restores Treg suppressive capacity}

We then wished to examine whether blockade of the PI3K/AKT/ Foxo1/3 pathway in Th1-Tregs would inhibit IFN $\gamma$ and restore their suppressive capacity (Fig 6). Tregs were stimulated in the presence of vehicle or IL-12 to generate Th1-Tregs, and 1 day later, a PI3K (LY294002) or an AKT1 inhibitor (MK2206) was added to the culture with subsequent examination of IFNG and TBX21 gene expression every $24 \mathrm{~h}$ for 3 days. As MK2206 can inhibit the three AKT isoforms, we used a concentration to inhibit only AKT1. Inhibition of either PI3K or AKT1 significantly decreased the expression of IFNG and TBX21 on Th1-Tregs (Fig 6A). The inhibition of IFN $\gamma$ was further confirmed at protein level, as incubation of Th1-Tregs with LY294002 or MK2206 significantly decreased the frequency of $\mathrm{IFN}^{+} \mathrm{Foxp}^{+}$Tregs, measured 4 days after activation (Fig 6B). While LY294002 abolished IFN $\gamma$ secretion almost completely, MK2206 inhibited it only 

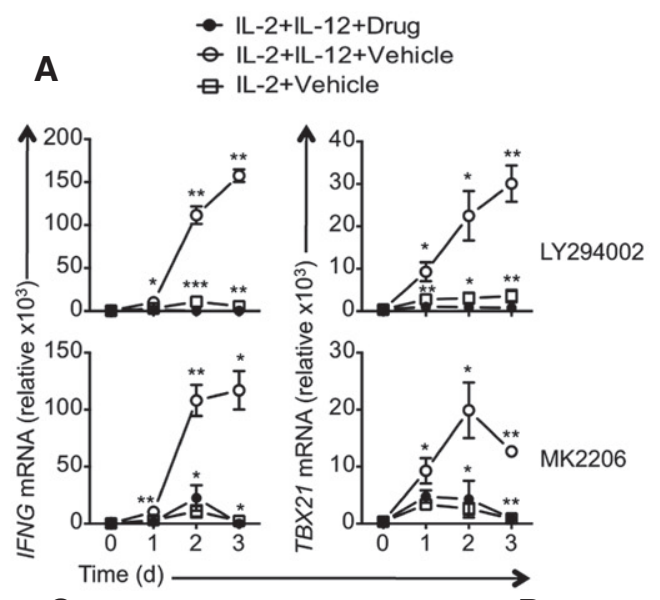

B
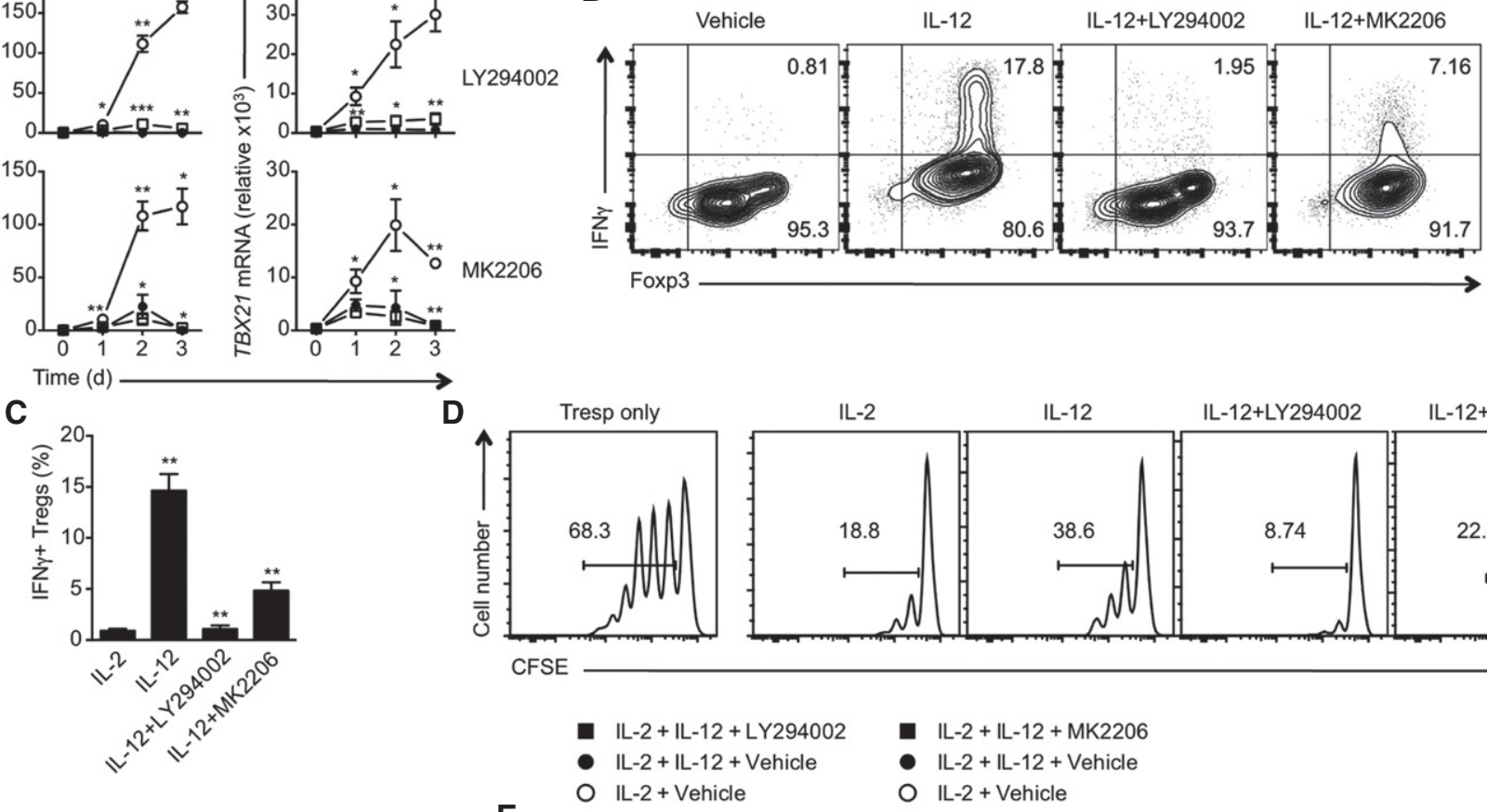

E

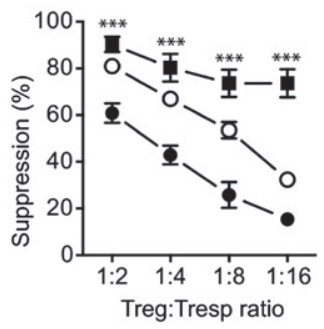

- $\mathrm{IL}-2+\mathrm{IL}-12+\mathrm{MK} 2206$

- $\mathrm{IL}-2+\mathrm{IL}-12+$ Vehicle

O IL-2 + Vehicle

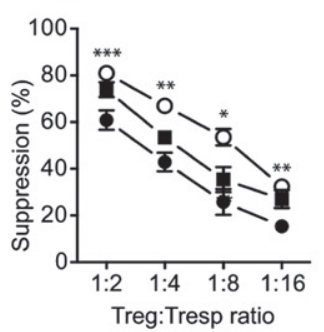

Figure 6. Blockade of the PIЗK/AKT/Foxo pathway in Th1-Tregs inhibits IFN $\gamma$ secretion and restores their suppressive function.

A Sorted $\mathrm{CD}^{+}{ }^{+} \mathrm{CD} 25^{\text {hi }} \mathrm{CD} 127^{-}$Tregs were stimulated in the presence of vehicle (white squares) and IL- 12 either alone (white circles) or in the presence of LY294002 or MK2206 (black circles) for 3 days, and IFNG and TBX21 gene expression was examined every 24 h. Statistical analysis of $n=8$ independent experiments performed.

B Representative example of the frequency of IFN $\gamma^{+} \mathrm{Foxp}^{+}$cells in Tregs stimulated in the presence of vehicle or IL-12 either alone or in the presence of LY294002 or MK2206 for 3 days after a 4 h stimulation with PMA and ionomycin.

C Statistical analysis of the frequency of IFN $\gamma^{+}$Foxp $^{+}$cells in Tregs stimulated as in (B) of $n=8$ experiments performed

D Proliferation measured at day 4 of CFSE-labeled responder T cells alone (left histogram) or co-cultured with Tregs that had been pre-incubated with either vehicle or IL-12 alone or with LY294002 or MK2206 at a 1:2 Treg:Tresp ratio.

E Statistical analysis of the percentage of suppression as in (D) at different Treg:Tresp ratios of $n=6$ experiments performed.

Data information: Data are presented as mean \pm s.e.m. ${ }^{*} p<0.05,{ }^{* *} p<0.005,{ }^{* *} p<0.0005$. Two-way ANOVA corrected for multiple comparisons with a Sidak test

performed for (A) and (E), and a one-way ANOVA with a Tukey's post-test for (C).

partially, suggesting that AKT1 blockade is not enough to completely inhibit the Th1-Treg phenotype (Fig 6C). Furthermore, when Th1-Tregs were pre-incubated with either LY294002 or MK2206, washed, and co-cultured with Tresp, they were able to efficiently suppress the proliferation of Tresp as compared to Th1-Tregs pre-treated with vehicle (Fig 6D). While AKT1 blockade improved suppression, but not to the suppressive capacity achieved by control Tregs, PI3K blockade fully restored suppression even above the suppressive capacity of control Tregs (Fig 6E). Furthermore, treatment of in vitro-derived Th1-Tregs with MK2206 and LY294002 decreased the frequency of proliferating cells and IL-2 secretion (Appendix Fig S4). These data suggest that the PI3K/AKT/Foxo1/3 pathway activation is necessary to maintain the Th1-Treg phenotype and function.

\section{PI3K/AKT/Foxo1/3 pathway blockade in Tregs from MS patients inhibits IFN $\gamma$ secretion and restores their suppressive ability}

Th1-Tregs have been observed in vivo in several human pathologies such as type 1 diabetes [9] and hepatitis C virus infection [33]. In 
this respect, we have previously shown that RRMS patients show a significant increase in the frequency of Th1-Tregs ex vivo [6]; thus, we used Tregs from patients with RRMS as an in vivo Th1-Treg model to examine the hypothesis that in vivo-generated Th1-Tregs display an activated PI3K/AKT/Foxo1/3 pathway and its blockade would reduce IFN $\gamma$ secretion and restore their suppressive ability. We first confirmed the upregulation of IFNG and TBX21 in ex vivosorted Tregs from RRMS as compared to healthy controls (HC) [6] in our group of patients (Fig EV4). We then examined the expression of the differentially expressed genes in IFN $\gamma^{+}$Tregs (Fig 1) in ex vivo-isolated Tregs from RRMS and compared them to $\mathrm{HC}$ (Fig 7A). A significant downregulation of PTEN, AKT3, and FOXO3 was observed while no differences were observed in the expression of AKT1, AKT2, or FOXO1. It was of interest that decreases in AKT3, $P T E N$, and FOXO3 were confirmed at protein level in Tregs from patients, demonstrating that these RRMS patients express less levels of the three proteins as compared to paired controls (Fig 7B). Furthermore, the phosphorylation status of AKT, Foxo1, and Foxo3 was significantly increased in ex vivo-isolated Tregs from RRMS patients as compared to healthy donors (Fig 7C), suggesting that the PI3K/AKT/Foxo1/3 pathway was activated in vivo in RRMS patients.

As blockade of the PI3K/AKT/Foxo1/3 pathway restores Th1Treg phenotype and function (Fig 6), we hypothesized that PI3K or AKT1 inhibition in RRMS Tregs would decrease the frequency of IFN $\gamma^{+}$Foxp $3^{+}$Tregs and restore their suppressive capacity. Sorted Tregs from RRMS patients and healthy controls were stimulated with anti-CD3 and anti-CD28 in the presence of either vehicle, LY294002, or MK2206 for 3 days. Intracellular staining revealed that inhibition of both PI3K and AKT1 on Tregs from RRMS patients inhibited IFN $\gamma$ secretion while not having any effect on Tregs from controls (Fig 7D and E). More importantly, when Tregs from RRMS patients were pre-incubated with LY294002 or MK2206, they were able to efficiently suppress Tresp proliferation, with LY294002 being able to restore Treg suppressive capacity above the suppressive ability of Tregs from healthy control subjects. PI3K or AKT1 blockade did not have any effect in the suppressive capacity of Tregs from healthy donors (Fig 7F and G).

Thus, our data indicates that the Th1-Treg phenotype observed in Tregs from RRMS is, at least in part, due to an in vivo activation of the PI3K/AKT/Foxo1/3 pathway, that can be corrected by PI3K or AKT1 blockade.

\section{Discussion}

Here, we describe the signaling pathway components involved in the reprogramming of human Tregs into a Th1-Treg phenotype. Using a genome-wide expression approach, differentially expressed genes in $\mathrm{IFN}^{+}$Tregs as compared to IFN $\gamma^{-}$Tregs were clustered into signaling pathways. This strategy led to the identification of the PI3K/AKT/Foxol/3 signaling pathway as central for Th1-Treg generation. Several important molecules from this pathway were differentially expressed not only in ex vivo IFN $\gamma^{+}$Th1-Tregs, but also during in vitro Th1-Treg reprogramming by IL-12, suggesting that the pathway is important for Th1Treg generation and maintenance. Moreover, activation of the PI3K/AKT/Foxo1/3 pathway led to Treg reprogramming to Th1like Tregs, while blockade of the pathway on Th1-like Tregs reversed their phenotype and Tregs reacquired their suppressive capacity. Finally, specific targeting of the PI3K/AKT/Foxo1/3 pathway decreased IFN $\gamma^{+}$Treg expression and restored function of defective Tregs in patients with MS. These data highlight a new potential therapeutic strategy for the treatment of autoimmune and viral disease.

The PI3K/AKT pathway is important for Treg development both in vitro [34] and in vivo [35], but its role in the phenotype, function, and/or stability of established Tregs is not well understood. In this regard, it has been shown that AKT phosphorylation is defective in Tregs as compared to Tresp after TCR stimulation and this diminished activation is necessary for the Tregs to efficiently perform their suppressive function [36]. In order to examine the role of the PI3K/AKT pathway in Th1-Treg generation, we demonstrated that in vitro-generated IL-12-driven Th1-Tregs exhibit an activated PI3K/AKT/Foxo1/3 pathway, in agreement with published data suggesting the activation of AKT by IL-12 [37]. Moreover, we induced the Th1-Treg phenotype not only by IL-12-driven AKT activation, but also by simply stimulating Tregs in the presence of PI3K or AKT1 activators, or PTEN or Foxo1/3 inhibitors, in agreement with recent data highlighting the importance of the PI3K pathway and PTEN on mice Treg stability [38,39]. In all cases, Tregs upregulated IFN $\gamma$ protein expression and TBX21 expression and were defective in suppressive capacity as compared to vehicle-treated Tregs.

Factors downstream of AKT activation, particularly the transcription factors Foxo1 and Foxo3, also have been reported to have

Figure 7. Blockade of the PI3K/AKT/Foxo pathway in Tregs from RRMS patients inhibits the Th1-Treg phenotype and restores their suppressive ability.

A mRNA expression on ex vivo-isolated Tregs from MS patients and healthy controls $(n=15)$.

B Representative example of the expression of AKT3, Foxo3, and PTEN on ex vivo-sorted Tregs from MS patients (black open histogram) and HC (gray open histogram). Gray histograms represent isotype.

C Ex vivo expression of pAKT, pFoxo1, and pFoxo3 in Tregs from RRMS patients (black open histogram) and healthy controls (white open histograms). Gray histograms represent isotype.

D Representative example of the frequency of IFN $\gamma^{+} \mathrm{Foxp}^{+}{ }^{+}$Tregs isolated ex vivo from RRMS patients (lower row) and healthy controls (HC, upper row) and stimulated in the presence of vehicle (left histogram), LY294002 (middle histogram), or MK2206 (right histogram) for 3 days. Staining was performed after a 4-h stimulation with PMA and ionomycin.

E Statistical analysis of the frequency of IFN $\gamma^{+}$Foxp3 ${ }^{+}$Tregs in HC (white bars) and RRMS patients (black bars) stimulated in the presence of either vehicle, LY294002, or MK2206 $(n=10)$.

F Representative example of the proliferation of Tresp cells from HC (upper row) and RRMS patients (lower row) either alone (first column) or in co-culture with Tregs that had been previously incubated with either vehicle, LY294002, or MK2206 for $1 \mathrm{~h}$ and washed to remove drug traces.

G Statistical analysis of the percentage of suppression as in (F) at different Treg:Tresp ratios of $n=8$ experiments performed.

Data information: Data are presented as mean \pm s.e.m. ${ }^{\star} P<0.05,{ }^{\star \star} P<0.005$, ${ }^{\star \star \star} P<0.0005$. $t$-test was performed for (A) and two-way ANOVA corrected for multiple comparisons with a Sidak test for (E) and (G). 
important, but partially redundant roles in Treg development [40]. We observed that AKT activation by IL-12 stimulation of Tregs also phosphorylated Foxo1 and Foxo3 leading to exclusion from the nucleus, precluding modulation of their transcriptional targets Foxo1 is essential for Treg function, and in its absence, Tregs from mice spontaneously express IFN $\gamma$ [22]. In agreement with this
A
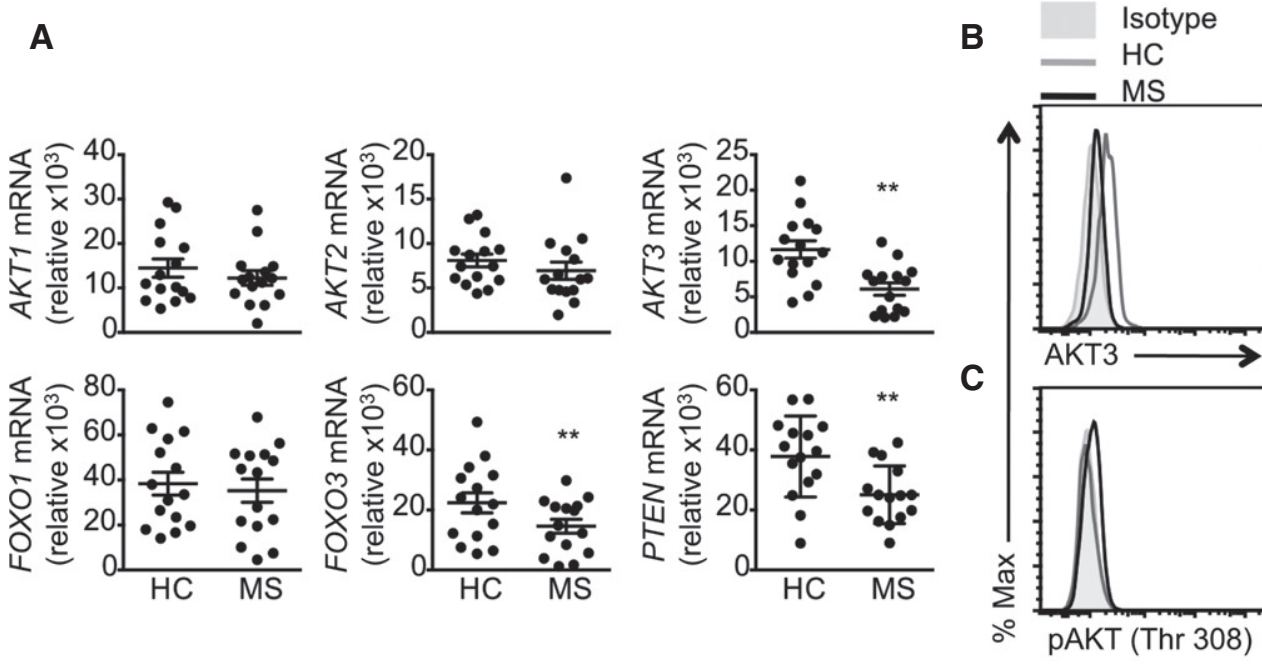

Isotype
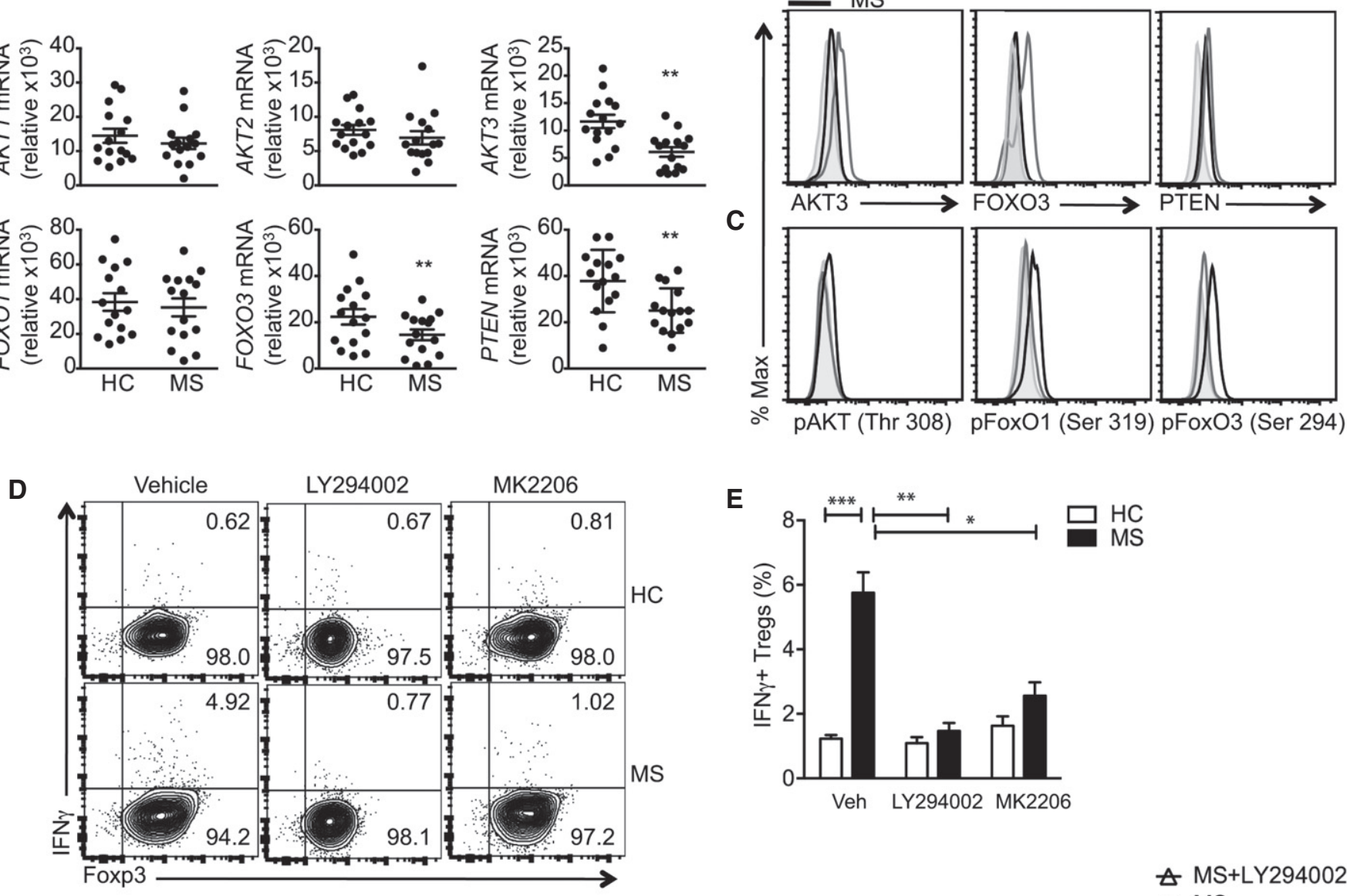

E

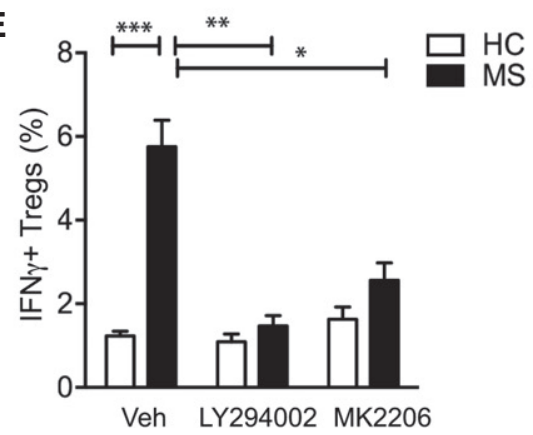

$\mathbf{F}$

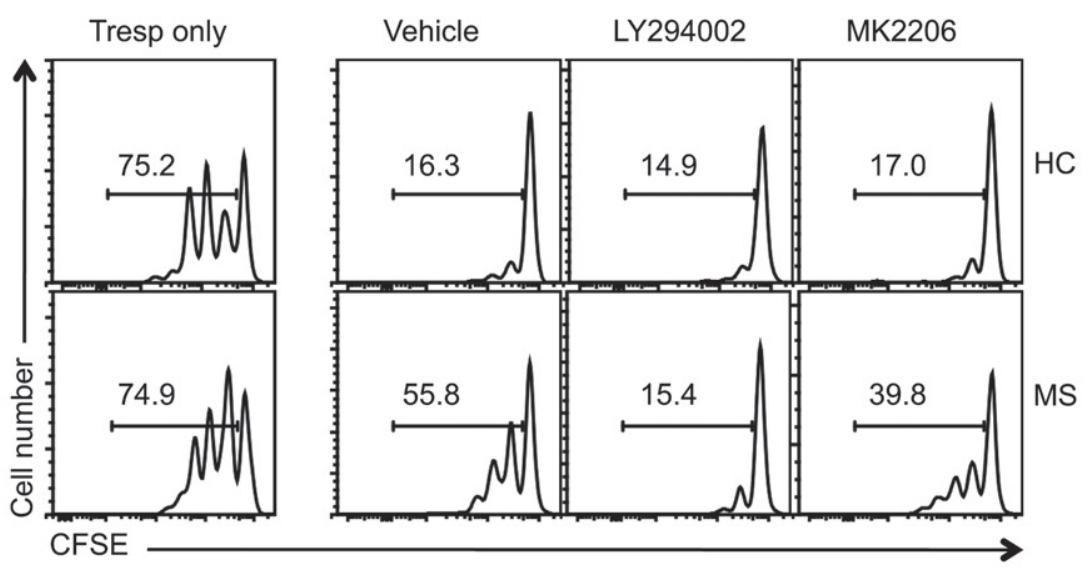

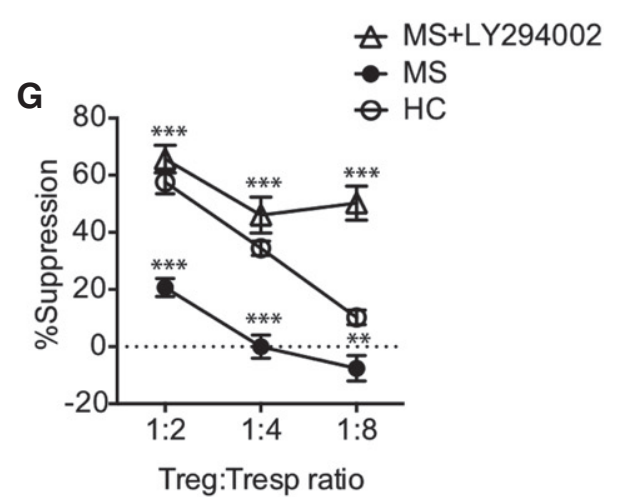

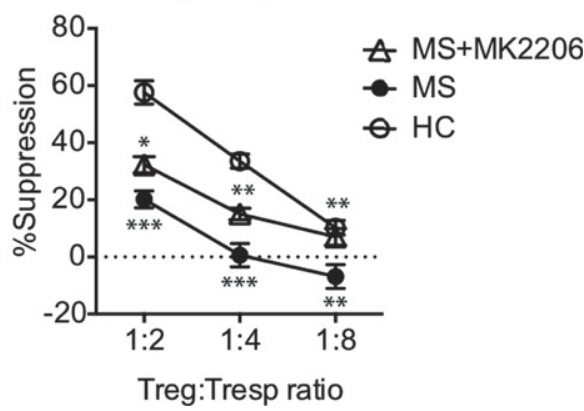

Figure 7. 
report, we observed that blocking both Foxo1 and Foxo3 with a synthetic chemical inhibitor was sufficient to induce IFN $\gamma$ expression by Tregs and decrease their suppressive capacity. Interestingly, the level of suppression inversely correlated with the frequency of Th1-Tregs in the co-culture, suggesting that IFN $\gamma$ is responsible, at least in part, for the defect in suppression observed, as previously suggested $[6,8]$. To further examine the role of each Foxo protein separately, we silenced both Foxo1 and Foxo3 in Tregs from healthy donors and observed that in both cases, the Tregs acquire a Th1 phenotype, confirming their overlapping functions in Th1-Treg generation.

While most of the reports in the literature examine global AKT expression and phosphorylation, gene expression data in Th1-Tregs demonstrated upregulation of AKT1 with downregulation of AKT2 and AKT3, prompting us to examine the role of the individual isoforms in Th1-Treg generation. Interestingly, we observed that AKT1 blockade greatly inhibits Th1-Treg generation, in agreement with previous reports by Levings and coworkers [36], while surprisingly, AKT3 silencing was sufficient to induce IFN $\gamma$ expression on Tregs. There are some examples where non-overlapping functions of AKT isoforms have been described [41-43], and interestingly, a recent paper has suggested a role for AKT3 in protecting mice from EAE [44]. The choice decision for activation of one or other isoform could be determined by the stimulus that is activating the pathway, or the specific cell type being examined.

PI3K/AKT are often dysregulated in autoimmune disease, and the expression of a constitutively active form of AKT leads to autoimmunity $[45,46]$. The importance of the PI3K/AKT/Foxo1/3 signaling pathway is also suggested by the fact that various murine models that demonstrate increased activity of the PI3K/AKT pathway signaling have an autoimmune phenotype (reviewed in [47]). In MS, there are likely several signaling pathways that lead to Th1Treg generation. The one described here can be activated by either the ex vivo downregulation of PTEN, the ex vivo increased phosphorylation of AKT, and/or the downregulation of AKT3. In relation to PTEN, it has been demonstrated that Tregs display increased levels of PTEN ex vivo, and these levels are maintained upon stimulation, in contrast to responder $\mathrm{T}$ cells, where it is downregulated rapidly after stimulation $[48,49]$. Furthermore, PTEN deficiency in rodent Tregs upregulates IFN $\gamma$ and these cells are not capable of restraining Th1 and Th responses in vivo [38,39].

Th1-Tregs have not only been observed in human autoimmune diseases such as MS and type 1 diabetes [6,9], but also during viral and bacterial infections [10,33], suggesting that the environmental cue that induces the Th1-like phenotype could be different in each disease setting. While the emergence of Th1-Tregs in acute infection could be a physiological protective mechanism during the adaptive phase of the immune response, the increased frequency of Th1Tregs in human autoimmune diseases is a potentially detrimental phenotype for the outcome of the disease. Nevertheless, modulation of the PI3K/AKT/Foxo1/3 pathway during these diseases could be a potential target for Treg function control.

Other signaling pathways are likely playing a role in Th1-Tregs generation (Appendix Table S1). Whether these pathways are acting at the same time and/or anatomical location, and their activation/ inhibition depends on different environmental cues needs further investigations. The fact that PI3K activation can be triggered by different receptor engagements supports the hypothesis that in humans, IFN $\gamma$ secretion and the acquisition of a Th1-Treg phenotype is a common "default" mechanism that dysfunctional Tregs adopt in several disease settings such as autoimmunity and acute infection. Nevertheless, these data identify novel targets for restoring the loss of regulatory $\mathrm{T}$-cell function in patients with autoimmune disease.

\section{Materials and Methods}

\section{Study subjects}

Peripheral blood was drawn from healthy individuals and 15 subjects with relapsing-remitting MS and their age- and sexmatched controls after informed consent and approval by the Institutional Review Board at Yale University. The patients were of ages of $44.0 \pm 4.1$ years, and all had relapsing-remitting disease with Kurtzke Expanded Disability Status Scale scores between 0 and 2.5. All patients $(n=15)$ were not treated with any immunomodulatory drugs. All experiments were performed conformed to the principles set out in the WMA Declaration of Helsinki and the Department of Health and Human Services Belmont Report.

\section{Cell culture reagents and antibodies}

Cells were cultured in RPMI 1640 media supplemented with $2 \mathrm{nM}$ L-glutamine, $5 \mathrm{mM} \mathrm{HEPES}$, and $100 \mathrm{U} / \mu \mathrm{g} / \mathrm{ml}$ penicillin/streptomycin (Biowhittaker, Walkersville, MD), $0.5 \mathrm{mM}$ sodium pyruvate, $0.05 \mathrm{mM}$ nonessential amino acids (Life Technologies, Rockville, $\mathrm{MD}$ ), and 5\% human $\mathrm{AB}$ serum (Gemini Bio-Products, Woodland, CA). The antibodies used for stimulation were anti-human CD3 (clone UCHT1) and anti-human CD28 (clone 28.2) (BD Biosciences, San Jose, CA) at $1 \mu \mathrm{g} / \mathrm{ml}$. Treg Inspector Beads (Miltenyi Biotec, Bergisch Gladbach, Germany) were used in suppression assays. IL-12 (R\&D Systems, Minneapolis, MN) was used at $20 \mathrm{ng} / \mathrm{ml}$. IL-2 was obtained through the AIDS Research and Reference Reagent Program, Division of AIDS, National Institute of Allergy and Infectious Diseases (NIAID), National Institutes of Health (NIH) and was used at $25 \mathrm{U} / \mathrm{ml}$.

The PTEN inhibitor SF1670 (Echelon Biosciences, Salt Lake City, UT) was used at $500 \mathrm{nM}$ [50]. The PI3K activator $740 \mathrm{Y}-\mathrm{P}$ (Tocris, Minneapolis, MN) was used at $20 \mu \mathrm{M}[51,52]$. The AKT activator SC-79 (EMD Millipore, Billerica, MA) was used at $4 \mu \mathrm{g} / \mathrm{ml}$ [53]. The Foxo1/Foxo3 inhibitor AS1842856 (EMD Millipore) was used at different concentrations ranging from $1 \mathrm{nM}$ to $50 \mathrm{nM}$, as previously published [54,55]. The PI3K inhibitor LY294002 (Invivogen, San Diego, CA) was used at $10 \mu \mathrm{M}$ [56-58]. MK2206 (SelleckChem, Pittsburgh, PA) was used at a concentration of $5 \mu \mathrm{M}$, based on similar published works performed in T cells and Tregs [59,60], and our own titration experiments. None of the small molecules used in this work induced significant cell death at the concentrations described above (Fig EV5).

\section{Cell isolation and FACS sorting of T-cell populations}

Peripheral blood mononuclear cells (PBMC) were isolated from healthy donors or patients with MS after informed consent was 
obtained in accordance with the Declaration of Helsinki by Ficoll Hypaque gradient centrifugation. Total $\mathrm{CD}^{+} \mathrm{T}$ cells were isolated by negative selection using $\mathrm{CD}^{+}{ }^{+} \mathrm{T}$-cell isolation kit (StemCell Technologies, Vancouver, BC) and stained for fluorescenceactivated cell sorting (FACS) with anti-CD25 (clone M-A251, BD Biosciences) and CD127 (clone eBioRDR5) from eBioscience (San Diego, CA). The Treg $\left(\mathrm{CD} 4{ }^{+} \mathrm{CD} 25^{\text {high }} \mathrm{CD} 127^{\text {neg }}\right)$ and responder T-cell (Tresp, $\mathrm{CD} 4{ }^{+} \mathrm{CD} 25^{\mathrm{dim} / \mathrm{low}} \mathrm{CD} 127^{+}$) populations were sorted on a FACS Aria (BD Biosciences).

\section{Cell activation and intracellular staining}

Cell populations were stimulated with $1 \mu \mathrm{g} / \mathrm{ml}$ plate-bound anti$\mathrm{CD} 3,1 \mu \mathrm{g} / \mathrm{ml}$ soluble anti-CD28, and $25 \mathrm{U} / \mathrm{ml}$ IL-2 in the presence or absence of IL-12. At day 3, cells were stimulated with $50 \mathrm{nM}$ phorbol-12-myristate-13-acetate (PMA) and $250 \mathrm{nM}$ ionomycin for $4 \mathrm{~h}$ in the presence of GolgiStop (BD Biosciences) and intracellular staining of IFN $\gamma$, and Foxp3 was performed with Foxp3 staining buffers (eBioscience) as per manufacturer's recommendations and the following antibodies: IFN $\gamma$ (clone 4S.B3) from Biolegend (San Diego, CA) and Foxp3 (clone PCH101) from eBioscience.

\section{Microarray sample preparation and analysis}

Tregs from healthy donors were sorted and stimulated with $50 \mathrm{ng}$ PMA and 250 ng ionomycin for $4 \mathrm{~h}$. IFN $\gamma$ detection kit (Miltenyi) was used to stain cytokine-secreting cells. Cells were resorted based on the expression of IFN $\gamma$. Samples were subjected to microarray analysis on an Affymetrix platform, and the data was quantilenormalized (Bioconductor Affy package) and log2-transformed. Quality control was performed using ArrayQualityMetrics [61], and arrays were preprocessed with RMA [62]. Differential expression was calculated using LIMMA package for single channel paired experimental design with Benjamini and Hochberg multiple testing adjustment [63]. Microarray analysis and description were carried out according to Minimum Information About a Microarray Experiment (MIAME) guidelines (GEO accession number GSE71274 [64]). Differentially expressed genes (fold change cutoff 1.5) were clustered into signaling pathways by Ingenuity Pathway Analysis (www.ingenuity.com/products/ipa) and sorted by BioRank. BioRank is a novel way to identify pathways and is complementary to the $P$-value enrichment score for each pathway, which is generated using the Fisher's exact enrichment test and primarily identifies significant overlap between your DEGs and the pathways. BioRank, besides identifying significant overlap between the DEGs and pathways, identifies cases where the DEGs from the dataset play an essential role in the pathway (www.ingenuity.com/products/ipa).

\section{Quantification of mRNA expression levels by RT-PCR}

RNA was isolated using QIAGEN RNeasy Micro Kit (QIAGEN, Valencia, CA), following manufacturer's guidelines and converted to cDNA by reverse transcription (RT) with random hexamers and Multiscribe RT (TQMN, Reverse Transcription Reagents; Applied Biosystems, Foster City, CA). For mRNA gene expression assays, probes were purchased from Applied Biosystems and the reactions were set up following manufacturer's guidelines and run on a StepOne Real-Time PCR System (Applied Biosystems). Values are represented as the difference in $C_{\mathrm{t}}$ values normalized to $\beta 2$-microglobulin for each sample as per the following formula: Relative RNA expression $=\left(2^{-\mathrm{d} C_{\mathrm{t}}}\right) \times 1,000$.

\section{Suppression assays}

For suppression of Th1-Tregs, Tregs were stimulated with anti-CD3 and anti-CD28 in the presence or absence of IL-12 for 2 days, washed, and either co-cultured with $10^{4}$ CFSE-labeled responder $\mathrm{CD} 4{ }^{+} \mathrm{CD} 25^{\mathrm{dim} /{ }^{\text {low }}} \mathrm{CD} 127^{+} \mathrm{T}$ cells at different Treg:Tresp ratios, or pre-incubated with different drugs for $1 \mathrm{~h}$ at $37^{\circ} \mathrm{C}$ and washed before putting them in co-culture. The stimulus used was Treg Inspector Beads (Miltenyi) at a 1:2 cell:bead ratio. At day 4, co-cultures were stained for viability with LIVE/DEAD stain kit (Invitrogen, Carlsbag, CA) and fixed using Foxp3 staining buffer (eBioscience), and proliferation of viable responder $\mathrm{T}$ cells was analyzed on a Fortessa flow cytometer (BD Biosciences). For some experiments, GolgiStop was added to the co-cultures for the last $6 \mathrm{~h}$ of incubation, and intracellular staining of IFN $\gamma$ and Foxp3 was performed after fixation and permeabilization.

\section{Gene silencing by lentiviral transduction}

Lentiviral particles encoding shRNAs were obtained from SigmaAldrich. $10^{5}$ sorted Tregs per well were stimulated with plate-bound anti-CD3 $(1 \mu \mathrm{g} / \mathrm{ml})$ and soluble anti-CD28 $(1 \mu \mathrm{g} / \mathrm{ml})$ for $24 \mathrm{~h}$ before transduction. Cells were then transduced with viral particles containing a vector expressing the indicated specific shRNA or a non-target shRNA as a control at a multiplicity of infection of 5. All constructions contained the coding sequence for GFP. After 5 days in culture, transduced cells were sorted based on GFP expression and the efficiency of gene silencing was determined by TaqMan real-time PCR and protein staining.

\section{Analysis of phosphorylation by FACS}

Treg cells were stimulated with $1 \mu \mathrm{g} / \mathrm{ml}$ anti-CD3 and $1 \mu \mathrm{g} / \mathrm{ml}$ anti-CD28 and $50 \mathrm{ng} / \mathrm{ml} \mathrm{IL-12}$ and fixed at different time points with fixation buffer (BD Biosciences), permeabilized with Perm Buffer III following manufacturer's recommendations, and stained with PE-labeled mouse anti-pAKT (pThr308) from BD Biosciences and pFoxo1 (pSer319) and pFoxo3 (pSer294) from Bioss. For stainings at $16 \mathrm{~h}$, Tregs were stimulated with $1 \mu \mathrm{g} / \mathrm{ml}$ anti-CD3 and $1 \mu \mathrm{g} / \mathrm{ml}$ anti-CD28 in the presence of $\mathrm{IL}-12$ at $50 \mathrm{ng} / \mathrm{ml}$ for $16 \mathrm{~h}$. For ex vivo stainings of MS and HC Tregs, ex vivo-sorted Tregs were fixed directly after sort with fixation buffer and stained as above. The specific time points selected to examine AKT, Foxo1, and Foxo3 phosphorylation were chosen based on preliminary kinetics experiments that were performed for a total of $50 \mathrm{~min}$.

\section{Statistics}

Statistical analysis was performed using GraphPad Prism (GraphPad Software). A standard paired two-tailed $t$-test was used for statistical analysis and a one-way ANOVA with a Tukey's post-test or a twoway ANOVA corrected for multiple comparisons with a Sidak test for more than two groups' comparisons; $P$-values of 0.05 or less were considered significant. Data were presented if not 
indicated elsewhere as mean \pm s.e.m. $\left({ }^{*} P<0.05,{ }^{* *} P<0.005\right.$, $\left.{ }^{* * *} P<0.0005\right)$. For correlation in Fig $3 \mathrm{~J}, r$ was calculated by Pearson correlation coefficient.

Expanded View for this article is available online.

\section{Acknowledgements}

The authors would like to thank Dr. L. Devine and Chao Wang for cell sorting and Dr. T. Bertrand for figure design and formatting. This work was supported by the Nancy Davis Foundation Junior Investigator Award and the Grant for Multiple Sclerosis Innovation (EMD Serono) to M.D.V. and the National Institutes of Health grants R01NS024247, P01AI03971, and P01NS038037 (D.A.H.).

\section{Author contributions}

AK, MdM, and A-SG performed experiments; MM analyzed microarray dataset, MD-V designed and performed experiments, analyzed data, wrote the manuscript, and supervised the study; and DAH supervised the study and wrote the manuscript.

\section{Conflict of interest}

The authors declare that they have no conflict of interest.

\section{References}

1. Fontenot JD, Gavin MA, Rudensky AY (2003) Foxp3 programs the development and function of $\mathrm{CD} 4^{+} \mathrm{CD} 25^{+}$regulatory T cells. Nat Immunol 4: $330-336$

2. Sakaguchi S, Miyara M, Costantino CM, Hafler DA (2010) FOXP3 ${ }^{+}$regulatory $T$ cells in the human immune system. Nat Reu Immunol 10: $490-500$

3. Hori S, Nomura T, Sakaguchi S (2003) Control of regulatory T cell development by the transcription factor Foxp3. Science 299: 1057-1061

4. Beriou G, Costantino CM, Ashley CW, Yang L, Kuchroo VK, Baecher-Allan C, Hafler DA (2009) IL-17-producing human peripheral regulatory T cells retain suppressive function. Blood 113: $4240-4249$

5. Du R, Zhao H, Yan F, Li H (2014) IL- $17^{+}$Foxp $^{+}$T cells: an intermediate differentiation stage between Th17 cells and regulatory T cells. J Leukoc Biol 96: $39-48$

6. Dominguez-Villar M, Baecher-Allan CM, Hafler DA (2011) Identification of T helper type 1-like, Foxp3 ${ }^{+}$regulatory T cells in human autoimmune disease. Nat Med 17: 673-675

7. Koch MA, Tucker-Heard G, Perdue NR, Killebrew JR, Urdahl KB, Campbell DJ (2009) The transcription factor T-bet controls regulatory T cell homeostasis and function during type 1 inflammation. Nat Immunol 10: 595-602

8. Lu LF, Boldin MP, Chaudhry A, Lin LL, Taganov KD, Hanada T, Yoshimura A, Baltimore D, Rudensky AY (2010) Function of miR-146a in controlling Treg cell-mediated regulation of Th1 responses. Cell 142: 914-929

9. McClymont SA, Putnam AL, Lee MR, Esensten JH, Liu W, Hulme MA, Hoffmuller U, Baron U, Olek S, Bluestone JA et al (2011) Plasticity of human regulatory $T$ cells in healthy subjects and patients with type 1 diabetes. J Immunol 186: 3918-3926

10. Oldenhove G, Bouladoux N, Wohlfert EA, Hall JA, Chou D, Dos Santos L, O'Brien S, Blank R, Lamb E, Natarajan S et al (2009) Decrease of Foxp3 ${ }^{+}$ Treg cell number and acquisition of effector cell phenotype during lethal infection. Immunity 31: 772-786
11. Kumar M, Putzki N, Limmroth V, Remus R, Lindemann M, Knop D, Mueller N, Hardt C, Kreuzfelder E, Grosse-Wilde H (2006) $\mathrm{CD}^{+} \mathrm{CD}^{2} 5^{+}$ FoxP3 ${ }^{+} \mathrm{T}$ lymphocytes fail to suppress myelin basic protein-induced proliferation in patients with multiple sclerosis. J Neuroimmunol 180: $178-184$

12. Venken K, Hellings N, Hensen K, Rummens JL, Medaer R, D'Hooghe MB, Dubois B, Raus J, Stinissen P (2006) Secondary progressive in contrast to relapsing-remitting multiple sclerosis patients show a normal $C D 4^{+}$ $\mathrm{CD} 25^{+}$regulatory T-cell function and FOXP3 expression. J Neurosci Res 83: $1432-1446$

13. Viglietta V, Baecher-Allan C, Weiner HL, Hafler DA (2004) Loss of functional suppression by $\mathrm{CD}^{+} \mathrm{CD}^{+} 5^{+}$regulatory $\mathrm{T}$ cells in patients with multiple sclerosis. J Exp Med 199: 971-979

14. Ward SG, Ley SC, MacPhee C, Cantrell DA (1992) Regulation of D-3 phosphoinositides during $T$ cell activation via the T cell antigen receptor/CD3 complex and CD2 antigens. Eur J Immunol 22: 45-49

15. Di Cristofano A, Kotsi P, Peng YF, Cordon-Cardo C, Elkon KB, Pandolfi PP (1999) Impaired Fas response and autoimmunity in Pten+/- mice. Science 285: 2122-2125

16. Migone TS, Rodig S, Cacalano NA, Berg M, Schreiber RD, Leonard WJ (1998) Functional cooperation of the interleukin-2 receptor beta chain and Jak1 in phosphatidylinositol 3-kinase recruitment and phosphorylation. Mol Cell Biol 18: 6416-6422

17. Schwindinger WF, Robishaw JD (2001) Heterotrimeric G-protein betagamma-dimers in growth and differentiation. Oncogene 20: 1653-1660

18. Knight ZA, Gonzalez B, Feldman ME, Zunder ER, Goldenberg DD, Williams O, Loewith R, Stokoe D, Balla A, Toth B et al (2006) A pharmacological map of the PI3-K family defines a role for p110alpha in insulin signaling. Cell 125: $733-747$

19. Okkenhaug K (2013) Signaling by the phosphoinositide 3-kinase family in immune cells. Annu Reu Immunol 31: 675-704

20. Stokoe D, Stephens LR, Copeland T, Gaffney PR, Reese CB, Painter GF, Holmes AB, McCormick F, Hawkins PT (1997) Dual role of phosphatidylinositol-3,4,5-trisphosphate in the activation of protein kinase B. Science 277: $567-570$

21. Brunet A, Bonni A, Zigmond MJ, Lin MZ, Juo P, Hu LS, Anderson MJ, Arden KC, Blenis J, Greenberg ME (1999) Akt promotes cell survival by phosphorylating and inhibiting a Forkhead transcription factor. Cell 96: 857-868

22. Ouyang W, Liao W, Luo CT, Yin N, Huse M, Kim MV, Peng M, Chan P, Ma Q, Mo Y et al (2012) Novel Foxo1-dependent transcriptional programs control T(reg) cell function. Nature 491: 554-559

23. Van Der Heide LP, Hoekman MF, Smidt MP (2004) The ins and outs of FoxO shuttling: mechanisms of FoxO translocation and transcriptional regulation. Biochem J 380: 297-309

24. Dijkers PF, Medema RH, Lammers JW, Koenderman L, Coffer PJ (2000) Expression of the pro-apoptotic $\mathrm{BCl}-2$ family member Bim is regulated by the forkhead transcription factor FKHR-L1. Curr Biol 10: $1201-1204$

25. Ouyang W, Beckett O, Flavell RA, Li MO (2009) An essential role of the Forkhead-box transcription factor Foxol in control of T cell homeostasis and tolerance. Immunity 30: 358-371

26. Kitamura T, Nakae J, Kitamura Y, Kido Y, Biggs WH III, Wright CV, White MF, Arden KC, Accili D (2002) The forkhead transcription factor Foxo1 links insulin signaling to $\mathrm{Pdx} 1$ regulation of pancreatic beta cell growth. J Clin Invest 110: 1839-1847

27. Zhao J, Brault JJ, Schild A, Cao P, Sandri M, Schiaffino S, Lecker SH, Goldberg AL (2007) FoxO3 coordinately activates protein degradation by the 
autophagic/lysosomal and proteasomal pathways in atrophying muscle cells. Cell Metab 6: 472-483

28. Birkenkamp KU, Essafi A, van der Vos KE, da Costa M, Hui RC, Holstege F, Koenderman L, Lam EW, Coffer PJ (2007) FOXO3a induces differentiation of Bcr-Abl-transformed cells through transcriptional down-regulation of Id1. J Biol Chem 282: 2211-2220

29. Rosivatz E, Matthews JG, McDonald NQ, Mulet X, Ho KK, Lossi N, Schmid AC, Mirabelli M, Pomeranz KM, Erneux C et al (2006) A small molecule inhibitor for phosphatase and tensin homologue deleted on chromosome 10 (PTEN). ACS Chem Biol 1: 780-790

30. Jo H, Mondal S, Tan D, Nagata E, Takizawa S, Sharma AK, Hou Q, Shanmugasundaram K, Prasad A, Tung JK et al (2012) Small molecule-induced cytosolic activation of protein kinase Akt rescues ischemia-elicited neuronal death. Proc Natl Acad Sci USA 109: 10581-10586

31. Derossi D, Williams EJ, Green PJ, Dunican DJ, Doherty P (1998) Stimulation of mitogenesis by a cell-permeable PI 3-kinase binding peptide. Biochem Biophys Res Commun 251: 148-152

32. Nagashima T, Shigematsu N, Maruki R, Urano Y, Tanaka H, Shimaya A, Shimokawa T, Shibasaki M (2010) Discovery of novel forkhead box 01 inhibitors for treating type 2 diabetes: improvement of fasting glycemia in diabetic db/db mice. Mol Pharmacol 78: 961-970

33. Piconese S, Timperi E, Pacella I, Schinzari V, Tripodo C, Rossi M, Guglielmo N, Mennini G, Grazi GL, Di Filippo S et al (2014) Human OX40 tunes the function of regulatory $T$ cells in tumor and nontumor areas of hepatitis $C$ virus-infected liver tissue. Hepatology 60: 1494-1507

34. Haxhinasto S, Mathis D, Benoist C (2008) The AKT-mTOR axis regulates de novo differentiation of $\mathrm{CD}^{+}{ }^{+}$Foxp3 $^{+}$cells. J Exp Med 205: $565-574$

35. Merkenschlager M, von Boehmer H (2010) PI3 kinase signalling blocks Foxp3 expression by sequestering Foxo factors. J Exp Med 207: $1347-1350$

36. Crellin NK, Garcia RV, Levings MK (2007) Altered activation of AKT is required for the suppressive function of human $\mathrm{CD}^{+} \mathrm{CD}_{2} 5^{+} \mathrm{T}$ regulatory cells. Blood 109: 2014-2022

37. Yoo JK, Cho JH, Lee SW, Sung YC (2002) IL-12 provides proliferation and survival signals to murine $\mathrm{CD}^{+}{ }^{+} \mathrm{T}$ cells through phosphatidylinositol 3kinase/Akt signaling pathway. J Immunol 169: 3637-3643

38. Huynh A, DuPage M, Priyadharshini B, Sage PT, Quiros J, Borges CM, Townamchai N, Gerriets VA, Rathmell JC, Sharpe AH et al (2015) Control of $\mathrm{PI}(3)$ kinase in Treg cells maintains homeostasis and lineage stability. Nat Immunol 16: 188-196

39. Shrestha S, Yang K, Guy C, Vogel P, Neale G, Chi H (2015) Treg cells require the phosphatase PTEN to restrain $\mathrm{TH} 1$ and TFH cell responses. Nat Immunol 16: 178-187

40. Kerdiles YM, Stone EL, Beisner DR, McGargill MA, Ch'en IL, Stockmann C, Katayama CD, Hedrick SM (2010) Foxo transcription factors control regulatory $\mathrm{T}$ cell development and function. Immunity 33: $890-904$

41. Gonzalez E, McGraw TE (2009) The Akt kinases: isoform specificity in metabolism and cancer. Cell Cycle 8: 2502-2508

42. Linnerth-Petrik NM, Santry LA, Petrik JJ, Wootton SK (2014) Opposing functions of Akt isoforms in lung tumor initiation and progression. PLOS ONE 9: e94595

43. Yu H, Littlewood T, Bennett M (2015) Akt isoforms in vascular disease. Vascul Pharmacol 71: 57-64

44. Tsiperson V, Gruber RC, Goldberg MF, Jordan A, Weinger JG, Macian F, Shafit-Zagardo B (2013) Suppression of inflammatory responses during myelin oligodendrocyte glycoprotein-induced experimental autoimmune encephalomyelitis is regulated by AKT3 signaling. J Immunol 190: $1528-1539$

45. Jones RG, Parsons M, Bonnard M, Chan VS, Yeh WC, Woodgett JR, Ohashi PS (2000) Protein kinase B regulates T lymphocyte survival, nuclear factor kappaB activation, and $\mathrm{BCl}-\mathrm{X}(\mathrm{L})$ levels in vivo. J Exp Med 191: $1721-1734$

46. Rathmell JC, Elstrom RL, Cinalli RM, Thompson CB (2003) Activated Akt promotes increased resting T cell size, CD28-independent T cell growth, and development of autoimmunity and lymphoma. Eur J Immunol 33: 2223-2232

47. Patel RK, Mohan C (2005) PI3K/AKT signaling and systemic autoimmunity. Immunol Res 31: 47-55

48. Zeiser R, Leveson-Gower DB, Zambricki EA, Kambham N, Beilhack A, Loh J, Hou JZ, Negrin RS (2008) Differential impact of mammalian target of rapamycin inhibition on $\mathrm{CD}^{+} \mathrm{CD}^{2} 5^{+} \mathrm{Foxp}^{+}$regulatory $\mathrm{T}$ cells compared with conventional CD4 + T cells. Blood 111: 453-462

49. Bensinger SJ, Walsh PT, Zhang J, Carroll M, Parsons R, Rathmell JC, Thompson CB, Burchill MA, Farrar MA, Turka LA (2004) Distinct IL-2 receptor signaling pattern in $\mathrm{CD}^{+} \mathrm{CD}_{2} 5^{+}$regulatory T cells. J Immunol 172: $5287-5296$

50. Li Y, Prasad A, Jia Y, Roy SG, Loison F, Mondal S, Kocjan P, Silberstein LE, Ding S, Luo HR (2011) Pretreatment with phosphatase and tensin homolog deleted on chromosome 10 (PTEN) inhibitor SF1670 augments the efficacy of granulocyte transfusion in a clinically relevant mouse model. Blood 117: 6702-6713

51. Anderson P, Gonzalez-Rey E (2010) Vasoactive intestinal peptide induces cell cycle arrest and regulatory functions in human T cells at multiple levels. Mol Cell Biol 30: 2537-2551

52. Bin BH, Bhin J, Yang SH, Choi DH, Park K, Shin DW, Lee AY, Hwang D, Cho EG, Lee TR (2014) Hyperosmotic stress reduces melanin production by altering melanosome formation. PLOS ONE 9: e105965

53. Su W, Wan Q, Han L, Huang J, Chen X, Chen G, Zheng SG, Liang D (2014) Doxycycline exerts multiple anti-allergy effects to attenuate murine allergic conjunctivitis and systemic anaphylaxis. Biochem Pharmacol 91: 359-368

54. Laine A, Martin B, Luka M, Mir L, Auffray C, Lucas B, Bismuth G, Charvet C (2015) Foxo1 is a T cell-intrinsic inhibitor of the RORgammat-Th17 program. J Immunol 195: 1791-1803

55. Trinite B, Chan CN, Lee CS, Mahajan S, Luo Y, Muesing MA, Folkvord JM, Pham M, Connick E, Levy DN (2014) Suppression of Foxol activity and down-modulation of CD62L (L-selectin) in HIV-1 infected resting CD4 T cells. PLOS ONE 9: e110719

56. Francois F, Klotman ME (2003) Phosphatidylinositol 3-kinase regulates human immunodeficiency virus type 1 replication following viral entry in primary $\mathrm{CD}^{+}{ }^{+} \mathrm{T}$ lymphocytes and macrophages. J Virol 77: 2539-2549

57. Garcon F, Patton DT, Emery JL, Hirsch E, Rottapel R, Sasaki T, Okkenhaug K (2008) CD28 provides T-cell costimulation and enhances PI3K activity at the immune synapse independently of its capacity to interact with the p85/p110 heterodimer. Blood 111: $1464-1471$

58. Sauer S, Bruno L, Hertweck A, Finlay D, Leleu M, Spivakov M, Knight ZA, Cobb BS, Cantrell D, O'Connor E et al (2008) T cell receptor signaling controls Foxp3 expression via PI3K, Akt, and mTOR. Proc Natl Acad Sci USA 105: $7797-7802$

59. Abu-Eid R, Samara RN, Ozbun L, Abdalla MY, Berzofsky JA, Friedman KM, Mkrtichyan M, Khleif SN (2014) Selective inhibition of regulatory 
T cells by targeting the PI3K-Akt pathway. Cancer Immunol Res 2: $1080-1089$

60. Simioni C, Neri LM, Tabellini G, Ricci F, Bressanin D, Chiarini F, Evangelisti C, Cani A, Tazzari PL, Melchionda F et al (2012) Cytotoxic activity of the novel Akt inhibitor, MK-2206, in T-cell acute lymphoblastic leukemia. Leukemia 26: $2336-2342$

61. Kauffmann A, Gentleman R, Huber W (2009) ArrayQualityMetrics-a bioconductor package for quality assessment of microarray data. Bioinformatics 25: $415-416$
62. Irizarry RA, Hobbs B, Collin F, Beazer-Barclay YD, Antonellis KJ, Scherf U, Speed TP (2003) Exploration, normalization, and summaries of high density oligonucleotide array probe level data. Biostatistics 4: 249-264

63. Ritchie ME, Phipson B, Wu D, Hu Y, Law CW, Shi W, Smyth GK (2015) Limma powers differential expression analyses for RNA-sequencing and microarray studies. Nucleic Acids Res 43: e47

64. Edgar R, Domrachev M, Lash AE (2002) Gene Expression Omnibus: NCBI gene expression and hybridization array data repository. Nucleic Acids Res 30: $207-210$ 